\title{
Molecular Design of Acrylates Containing Isocyanurate Moiety Undergoing Low Volume Shrinkage during Their Radical Photopolymerization
}

\author{
Shusuke Okamoto ${ }^{1}$, Toyofumi Shinozuka ${ }^{2}$, and Takeshi Endo ${ }^{*, 1}$
}

${ }^{1}$ Molecular Engineering Institute, Kyushu Institute of Technology, Sensui-cho 1-1, Tobata-ku, Kitakyushu, Fukuoka, 804-8550, Japan.

${ }^{2}$ Applied Materials Department Information Media Materials Development Laboratory R \& D Division, ADEKA Corporation, Higashiogu 7-2-34, Arakawa-ku, Tokyo, 116-8553, Japan.

*Corresponding author. E-mail address: endo.takeshi328@mail.kyutech.jp 


\section{Contents:}

\section{S1. Synthetic Procedures}

Scheme S1. Synthesis of diol 1

Scheme S2. Synthesis of acrylates $\mathbf{1 0}$ and $\mathbf{1 1}$

Scheme S3. Radical photopolymerization of acrylates $\mathbf{1 0}$ and $\mathbf{1 1}$

\section{S2. NMR data}

Figure S1. ${ }^{1} \mathrm{H}$ - and ${ }^{13} \mathrm{C}-\mathrm{NMR}$ spectra of diol $\mathbf{1}$

Figure S2. ${ }^{1} \mathrm{H}-{ }^{13} \mathrm{C}-\mathrm{NMR}$ spectrum of diol $\mathbf{1}$

Figure S3. ${ }^{1} \mathrm{H}$ - and ${ }^{13} \mathrm{C}$-NMR spectra of monool 2

Figure S4. ${ }^{1} \mathrm{H}$ - and ${ }^{13} \mathrm{C}-\mathrm{NMR}$ spectra of monool 3

Figure S5. ${ }^{1} \mathrm{H}-\mathrm{NMR}$ spectra of acrylate $\mathbf{4}$ and polymer $\mathbf{7}$

Figure S6. ${ }^{13} \mathrm{C}-\mathrm{NMR}$ spectra of acrylate 4 and polymer 7

Figure S7. ${ }^{1} \mathrm{H}-\mathrm{NMR}$ spectra of acrylate $\mathbf{5}$ and polymer $\mathbf{8}$

Figure S8. ${ }^{13}$ C-NMR spectra of acrylate $\mathbf{5}$ and polymer $\mathbf{8}$

Figure S9. ${ }^{1} \mathrm{H}-\mathrm{NMR}$ spectra of acrylate 6 and polymer 9

Figure S10. ${ }^{13} \mathrm{C}-\mathrm{NMR}$ spectra of acrylate 6 and polymer 9

Figure S11. ${ }^{1} \mathrm{H}^{1}{ }^{13} \mathrm{C}-\mathrm{NMR}$ spectrum of acrylate 6

Figure S12. ${ }^{1} \mathrm{H}$ - and ${ }^{13} \mathrm{C}-\mathrm{NMR}$ spectra of monool bearing a cyclohexane core

Figure S13. ${ }^{1} \mathrm{H}$ - and ${ }^{13} \mathrm{C}-\mathrm{NMR}$ spectra of monool bearing an alkyl moiety

Figure S14. ${ }^{1} \mathrm{H}-\mathrm{NMR}$ spectra of acrylate $\mathbf{1 0}$ and polymer $\mathbf{1 2}$

Figure S15. ${ }^{13} \mathrm{C}$-NMR spectra of acrylate $\mathbf{1 0}$ and polymer $\mathbf{1 2}$

Figure S16. ${ }^{1} \mathrm{H}-\mathrm{NMR}$ spectra of acrylate $\mathbf{1 1}$ and polymer $\mathbf{1 3}$

Figure S17. ${ }^{13} \mathrm{C}-\mathrm{NMR}$ spectra of acrylate $\mathbf{1 1}$ and polymer $\mathbf{1 3}$

Figure S18. Solid state ${ }^{13}$ C-NMR (CPMAS) spectrum of crosslinked polymer 14a 
Figure S19. Solid state ${ }^{13}$ C-NMR (CPMAS) spectra of crosslinked polymers 14b-c

\section{S3. IR data}

Figure S20. IR spectrum of diol 1

Figure S21. IR spectrum of monool 2

Figure S22. IR spectrum of monool 3

Figure S23. IR spectra of acrylate $\mathbf{4}$ and polymer $\mathbf{7}$

Figure S24. IR spectra of acrylate 5 and polymer $\mathbf{8}$

Figure S25. IR spectra of acrylate $\mathbf{6}$ and polymer $\mathbf{9}$

Figure S26. IR spectrum of monool bearing a cyclohexane core

Figure S27. IR spectrum of monool bearing an alkyl moiety

Figure S28. IR spectra of acrylate $\mathbf{1 0}$ and polymer $\mathbf{1 2}$

Figure S29. IR spectra of acrylate $\mathbf{1 1}$ and polymer $\mathbf{1 3}$

Figure S30. IR spectra of crosslinked polymers 14a-c

\section{S4. TG data}

Figure S31. TG-thermogram of polymer 7

Figure S32. TG-thermogram of polymer $\mathbf{8}$

Figure S33. TG-thermogram of polymer 9

Figure S34. TG-thermograms of crosslinked polymers 14a-c

\section{S5. DSC data}

Figure S35. DSC-thermograms of polymers 7-9

Figure S36. DSC-thermograms of crosslinked polymers 14a-c

\section{S6. Volume change during radical photo-copolymerization of TAI and 6}

Table S1. Results of volume change during radical photo-copolymerization of TAI and 6 


\section{S1. Synthetic Procedures}

\section{S1-1. Procedure for Synthesis of Diol 1 (Scheme S1)}<smiles>O=c1n(CCO)c(=O)n(CCO)c(=O)n1CCO</smiles>

THI

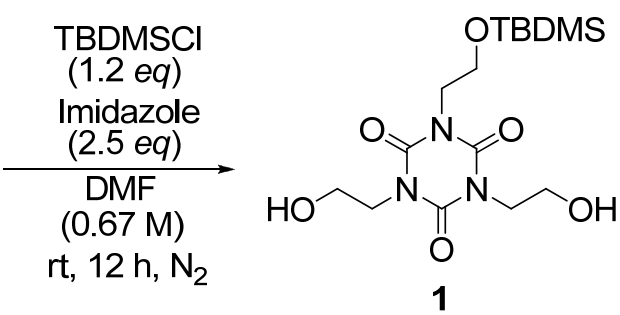

1

Scheme S1. Synthesis of diol 1

Synthesis of 1-(2-(tert-butyldimethylsilyloxy)ethyl)-3,5-bis(2-hydroxyethyl)-1,3,5triazinane-2,4,6-trione (1)

Tris(2-hydroxyethyl) isocyanurate (THI) $(15.7 \mathrm{~g} ; 60.0 \mathrm{mmol})$, imidazole (10.2 g; 150 mmol) and DMF (50 mL) were added to a $200 \mathrm{~mL}$ flask, and this reaction system was replaced under nitrogen atmosphere. After a solution of tert-butyl dimethyl silyl chloride (10.9 $\mathrm{g} ; 72.0 \mathrm{mmol})$ in DMF $(40 \mathrm{~mL})$ was dropwise added to this suspension in an ice bath, the reaction mixture was stirred at room temperature for 12 hours. The reaction was quenched by adding of $5 \%$ sodium carbonate aquation $(20 \mathrm{~mL})$. The resulting mixture was extracted from ethyl acetate $(200 \mathrm{~mL})$ twice, the obtained organic layer was washed with $5 \%$ sodium carbonate aquation $(200 \mathrm{~mL})$ and was dried over sodium sulfate for 2 hours, was filtrated with a suction. The obtained filtrate was concentrated under reduced pressure. The resulting residue was isolated by a silica gel chromatography (eluent : hexane/ethyl acetate $=2 / 1 \rightarrow 1 / 1 \rightarrow 1 / 4)$ to afford diol 1 as a white solid $(7.90 \mathrm{~g} ; 21.1 \mathrm{mmol}$; $35 \%$ ); m.p. $=70.2{ }^{\circ} \mathrm{C}-72.1{ }^{\circ} \mathrm{C} ;{ }^{1} \mathrm{H}-\mathrm{NMR}\left(400 \mathrm{MHz}, \mathrm{DMSO}-d_{6}\right.$, at r.t., ppm) $\delta 4.76(\mathrm{t}, J=$ $6.0 \mathrm{~Hz}, 2 \mathrm{H}), 3.86(\mathrm{t}, J=6.4 \mathrm{~Hz}, 2 \mathrm{H}), 3.81(\mathrm{t}, J=6.4 \mathrm{~Hz}, 4 \mathrm{H}), 3.70(\mathrm{t}, J=6.4 \mathrm{~Hz}, 2 \mathrm{H})$, $3.50(\mathrm{dt}, J=6.0,6.4 \mathrm{~Hz}, 4 \mathrm{H}), 0.82(\mathrm{~s}, 9 \mathrm{H}),-0.01(\mathrm{~s}, 6 \mathrm{H}) ;{ }^{13} \mathrm{C}-\mathrm{NMR}(100 \mathrm{MHz}$, DMSO$d_{6}$, at r.t., ppm) $\delta 148.98,58.98,57.49,44.23,43.66,25.66,17.84,-5.62$; IR (ATR, $\left.\mathrm{cm}^{-1}\right)$ 
$v$ 3249, 2936, 2930, 2861, 2858, 1675, 1448, 1354, 1322, 1253, 1192, 1094, 991, 913, 836, 773; HRMS m/z: $[\mathrm{M}+\mathrm{H}]^{+}$Calcd for $\left[\mathrm{C}_{15} \mathrm{H}_{30} \mathrm{~N}_{3} \mathrm{O}_{6} \mathrm{Si}_{1}\right]^{+}$376.19039; Found 376.19058.

S1-2. Procedure for Synthesis of Acrylates 10 and 11 (Scheme S2)<smiles>OC1CC(O)CC(O)C1</smiles>

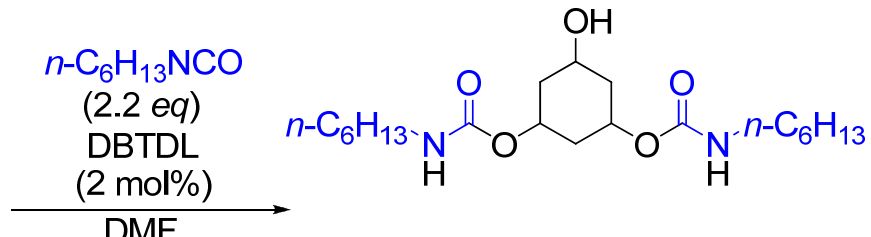<smiles>OCC(CO)(CO)CO</smiles>
$(1.0 \mathrm{M})$ $60^{\circ} \mathrm{C}, 16 \mathrm{~h}, \mathrm{~N}_{2}$

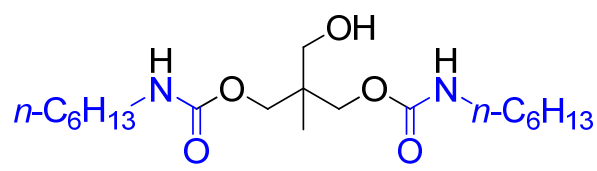

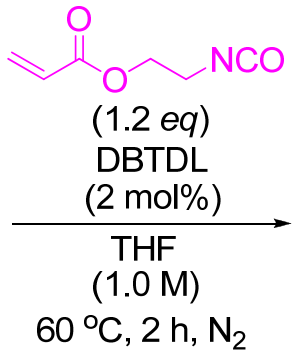

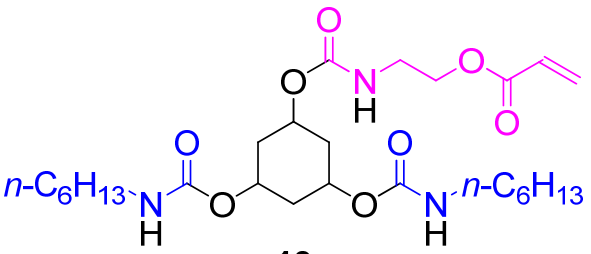

10

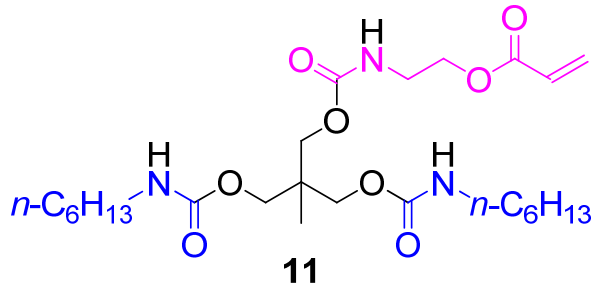

Scheme S2. Synthesis of acrylates $\mathbf{1 0}$ and $\mathbf{1 1}$

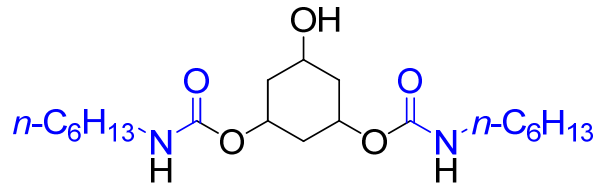

\section{Synthesis of 5-hydroxycyclohexane-1,3-diyl bis(hexylcarbamate)}

Typical procedure: 1,3,5-cyclohexane triol (1.32 g; $10.0 \mathrm{mmol})$ and DBDTL (126 mg; $0.200 \mathrm{mmol})$ were dissolved in DMF $(10 \mathrm{~mL})$ under nitrogen atmosphere. $n$-Hexyl isocyanate $(2.80 \mathrm{~g} ; 22.0 \mathrm{mmol})$ was added to the prepared solution via a syringe, and the reaction system was heated at $60{ }^{\circ} \mathrm{C}$ for 16 hours. The reaction solution was concentrated under reduced pressure, and the resulting crude was isolated by a silica gel column 
chromatography (eluent : hexane/ethyl acetate $=1 / 1 \rightarrow 1 / 2$ ) to afford the desired monool as a white solid $(1.69 \mathrm{~g} ; 4.37 \mathrm{mmol} ; 44 \%)$; m.p. $=105.8{ }^{\circ} \mathrm{C}-109.4{ }^{\circ} \mathrm{C} ;{ }^{1} \mathrm{H}-\mathrm{NMR}(400 \mathrm{MHz}$, DMSO- $d_{6}$, at r.t., ppm) $\delta 7.06(\mathrm{t}, J=5.8 \mathrm{~Hz}, 2 \mathrm{H}), 6.96(\mathrm{t}, J=5.6 \mathrm{~Hz}, 2 \mathrm{H}), 4.89-4.75(\mathrm{~m}$, $3 \mathrm{H}), 4.51-4.43(\mathrm{~m}, 2 \mathrm{H}), 4.07(\mathrm{~s}, 1 \mathrm{H}), 3.55-3.49(\mathrm{~m}, 1 \mathrm{H}), 2.92(\mathrm{dt}, J=6.4,6.8 \mathrm{~Hz}, 4 \mathrm{H})$, 2.16-2.06 (m, 2H), $1.85(\mathrm{~d}, J=12.8 \mathrm{~Hz}, 1 \mathrm{H}), 1.40-1.08(\mathrm{~m}, 19 \mathrm{H}), 0.84(\mathrm{t}, J=6.8 \mathrm{~Hz}$, $6 \mathrm{H}) ;{ }^{13} \mathrm{C}-\mathrm{NMR}(100 \mathrm{MHz}, \mathrm{DMSO}-d 6$, at r.t., ppm) $\delta 156.14,156.02,67.61,64.34,64.01$, $41.38,40.69,38.38,38.12,31.51,29.92,29.87,26.45,22.60,14.42 ; \mathrm{IR}\left(\mathrm{ATR}, \mathrm{cm}^{-1}\right) v$ $3514,3325,2939,2927,2856,1693,1660,1533,1466,1246,1228,1128,1070,1014$, 891, 775. HRMS $m / z$ : $[\mathrm{M}+\mathrm{Na}]^{+}$Calcd for $\left[\mathrm{C}_{20} \mathrm{H}_{38} \mathrm{~N}_{2} \mathrm{Na}_{1} \mathrm{O}_{5}\right]^{+}$409.26784; Found 409.26850.

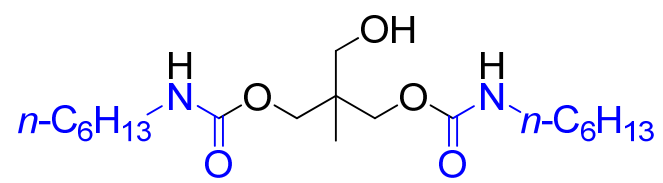

\section{Synthesis of 2-(hydroxymethyl)-2-methylpropane-1,3-diyl bis(hexylcarbamate)}

According to the synthesis procedure of 5-hydroxycyclohexane-1,3-diyl bis(hexylcarbamate), the reaction of trimethyloyl ethane $(1.20 \mathrm{~g} ; 10.0 \mathrm{mmol})$ with $n$-hexyl isocyanate $(2.80 \mathrm{~g} ; 22.0 \mathrm{mmol})$ in the presence of DBDTL (126 mg; $0.200 \mathrm{mmol})$ was performed in DMF (10 mL) under nitrogen atmosphere. After 16 hours, the resulting crude was isolated by a silica gel column chromatography (eluent : hexane/ethyl acetate $=2 / 1 \rightarrow 1 / 1)$ to afford the desired monool as a colorless liquid $(1.98 \mathrm{~g} ; 5.29 \mathrm{mmol}$; $53 \%) ;{ }^{1} \mathrm{H}-\mathrm{NMR}\left(400 \mathrm{MHz}, \mathrm{DMSO}-d_{6}\right.$, at r.t., ppm), $\delta 7.05(\mathrm{t}, J=5.8 \mathrm{~Hz}, 2 \mathrm{H}), 4.58(\mathrm{t}, J$ $=4.8 \mathrm{~Hz}, 1 \mathrm{H}), 3.79(\mathrm{~s}, 4 \mathrm{H}), 3.24(\mathrm{~d}, J=4.4 \mathrm{~Hz}, 1 \mathrm{H}), 2.92(\mathrm{dt}, J=6.0,6.8 \mathrm{~Hz}, 4 \mathrm{H}), 1.37-$ $1.22(\mathrm{~m}, 16 \mathrm{H}), 0.86-0.81(\mathrm{~m}, 9 \mathrm{H}) ;{ }^{13} \mathrm{C}-\mathrm{NMR}(100 \mathrm{MHz}, \mathrm{DMSO}-d 6$, at r.t., ppm) $\delta 156.85$, 66.02, 63.64, 40.68, 31.47, 29.86, 26.41, 22.55, 16.89, 14.39; IR (ATR, $\left.\mathrm{cm}^{-1}\right)$ v 3317, 2927, 2856, 1686, 1533, 1458, 1246, 1142, 1041, 1012, 775; HRMS $m / z:[\mathrm{M}+\mathrm{Na}]^{+} \mathrm{Calcd}$ 
for $\left[\mathrm{C}_{19} \mathrm{H}_{38} \mathrm{~N}_{2} \mathrm{Na}_{1} \mathrm{O}_{5}\right]^{+}$397.26784; Found 397.26833.

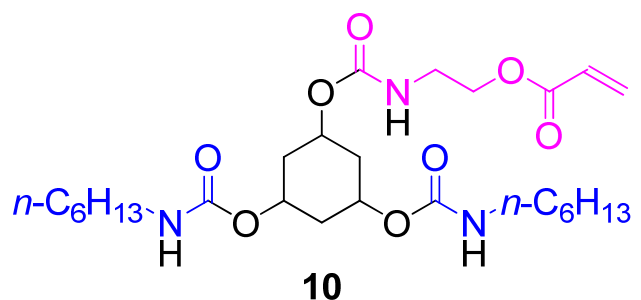

Synthesis of 2-((3,5-bis(hexylcarbamoyloxy)cyclohexyloxy)carbonylamino)ethyl acrylate (10)

According to the synthetic procedure of $\mathbf{6}$, the reaction of 5-hydroxycyclohexane-1,3diyl bis(hexylcarbamate) (773 mg; $2.00 \mathrm{mmol}$ ) with 2-isocyanaterthyl acrylate (339 $\mathrm{mg}$; $2.40 \mathrm{mmol})$ in the presence of DBTDL $(25.2 \mathrm{mg}$; $0.0400 \mathrm{mmol})$ was performed. This resulting solution was concentrated under reduced pressure, and the obtained residue was isolated by a silica gel column chromatography (eluent $:$ hexane/ethyl acetate $=1 / 1 \rightarrow 1 / 2$ ) to afford acrylate $\mathbf{1 0}$ as a colorless liquid (968 mg; $1.83 \mathrm{mmol} ; 92 \%) ;{ }^{1} \mathrm{H}-\mathrm{NMR}(400 \mathrm{MHz}$, DMSO- $d_{6}$, at r.t., ppm) $\delta 7.37(\mathrm{t}, J=5.6 \mathrm{~Hz}, 1 \mathrm{H}), 7.30(\mathrm{t}, J=5.6 \mathrm{~Hz}, 1 \mathrm{H}), 7.09(\mathrm{t}, J=5.6$ $\mathrm{Hz}, 2 \mathrm{H}), 7.04(\mathrm{t}, J=5.6 \mathrm{~Hz}, 2 \mathrm{H}), 6.38(\mathrm{~d}, J=17.6 \mathrm{~Hz}, 1 \mathrm{H}), 6.18-6.10(\mathrm{~m}, 1 \mathrm{H}), 5.93-5.89$ $(\mathrm{m}, 1 \mathrm{H}), 5.03-5.01(\mathrm{~m}, 1 \mathrm{H}), 4.79-4.74(\mathrm{~m}, 1 \mathrm{H}), 4.59-4.53(\mathrm{~m}, 2 \mathrm{H}), 4.08(\mathrm{t}, J=5.6 \mathrm{~Hz}$ $2 \mathrm{H}), 3.26-3.21(\mathrm{~m}, 2 \mathrm{H}), 2.92(\mathrm{dt}, J=6.0,6.4 \mathrm{~Hz}, 4 \mathrm{H}), 2.19-2.17(\mathrm{~m}, 2 \mathrm{H}), 1.95-1.92(\mathrm{~m}$, $1 \mathrm{H}), 1.54(\mathrm{t}, J=10.8 \mathrm{~Hz}, 1 \mathrm{H}), 1.37-1.21(\mathrm{~m}, 18 \mathrm{H}), 0.83(\mathrm{t}, J=6.8 \mathrm{~Hz}, 6 \mathrm{H}) ;{ }^{13} \mathrm{C}-\mathrm{NMR}$ (100 MHz, $\mathrm{CDCl}_{3}$, at r.t., ppm) $\delta 166.20,166.17,155.78,155.61,155.50,131.58,128.13$, $128.08,69.02,67.96,67.89,67.42,63.70,41.12,40.24,40.11,37.51,35.64,31.57,30.03$, 26.52, 22.67, 14.14; IR (ATR, $\left.\mathrm{cm}^{-1}\right) v$ 3319, 2941, 2927, 2856, 1693, 1522, 1456, 1408, 1240, 1192, 1132, 1011, 985, 781, 775; HRMS m/z: $[\mathrm{M}+\mathrm{Na}]^{+}$Calcd for $\left[\mathrm{C}_{26} \mathrm{H}_{45} \mathrm{~N}_{3} \mathrm{Na}_{1} \mathrm{O}_{8}\right]^{+}$550.31043; Found 550.30937. 


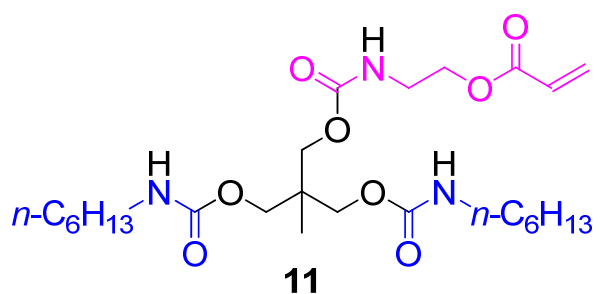

\section{Synthesis of 7-((hexylcarbamoyloxy)methyl)-7-methyl-4,10-dioxo-5,9-dioxa-3,11-}

\section{diazaheptadecyl acrylate (11)}

According to the synthetic procedure of $\mathbf{6}$, the reaction of 2-(hydroxymethyl)-2methylpropane-1,3-diyl bis(hexylcarbamate $) \quad(749 \quad \mathrm{mg} ; \quad 2.00 \quad \mathrm{mmol}) \quad$ with $\quad 2$ isocyanaterthyl acrylate $(339 \mathrm{mg} ; 2.40 \mathrm{mmol})$ in the presence of DBTDL (25.2 $\mathrm{mg}$; $0.0400 \mathrm{mmol}$ ) was performed. This resulting solution was concentrated under reduced pressure, and the obtained residue was isolated by a silica gel column chromatography (eluent : hexane/ethyl acetate $=2 / 1 \rightarrow 1 / 1)$ to afford acrylate $\mathbf{1 1}$ as a colorless liquid (951 mg; $1.84 \mathrm{mmol} ; 92 \%) ;{ }^{1} \mathrm{H}-\mathrm{NMR}\left(400 \mathrm{MHz}, \mathrm{DMSO}-d_{6}\right.$, at r.t., ppm) $\delta 7.34$ (t, $J=5.4 \mathrm{~Hz}$, $1 \mathrm{H}), 7.10(\mathrm{t}, J=5.2 \mathrm{~Hz}, 2 \mathrm{H}), 6.33(\mathrm{dd}, J=1.6,17.2 \mathrm{~Hz}, 1 \mathrm{H}), 6.14(\mathrm{dd}, J=10.4,17.2 \mathrm{~Hz}$, 1H), $5.93(\mathrm{dd}, J=1.2,10.8 \mathrm{~Hz}, 1 \mathrm{H}), 4.09(\mathrm{t}, J=5.4 \mathrm{~Hz}, 2 \mathrm{H}), 3.85-3.79(\mathrm{~m}, 6 \mathrm{H}), 3.24(\mathrm{dt}$, $J=5.2,6.0 \mathrm{~Hz}, 2 \mathrm{H}), 2.93(\mathrm{q}, J=6.4 \mathrm{~Hz}, 4 \mathrm{H}), 1.36-1.22(\mathrm{~m}, 16 \mathrm{H}), 0.86-0.82(\mathrm{~m}, 9 \mathrm{H})$; ${ }^{13} \mathrm{C}-\mathrm{NMR}\left(100 \mathrm{MHz}, \mathrm{CDCl}_{3}\right.$, at r.t., ppm) $\delta 166.11,156.73,156.43,131.50,128.00,66.55$, $66.17,63.54,41.79,41.12,40.15,38.90,31.55,29.90,26.54,26.44,22.61,22.58,16.88$, 16.71, 14.06; IR (ATR, $\left.\mathrm{cm}^{-1}\right) v$ 3317, 2937, 2926, 2856, 1693, 1529, 1466, 1408, 1244 , 1188, 1144, 1036, 810, 775; HRMS m/z: $[\mathrm{M}+\mathrm{Na}]^{+} \mathrm{Calcd}$ for $\left[\mathrm{C}_{25} \mathrm{H}_{45} \mathrm{~N}_{3} \mathrm{Na}_{1} \mathrm{O}_{8}\right]^{+}$ 538.31043; Found 538.31143. 


\section{S1-3. Procedure for Radical Photopolymerization of Acrylates 10 and 11 (Scheme}

S3)

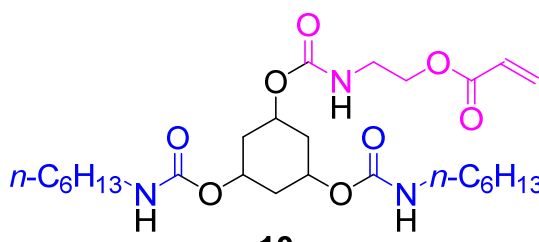

10

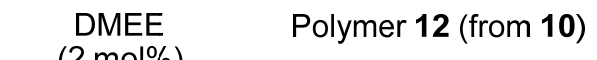

Polymer 13 (from 11)

Scheme S3. Radical photopolymerization of acrylates $\mathbf{1 0}$ and $\mathbf{1 1}$

\section{Radical photopolymerization of acrylate $\mathbf{1 0}$}

According to the typical procedure for the synthesis of $\mathbf{7}$, radical photopolymerization of $10(258 \mathrm{mg}$; $0.500 \mathrm{mmol})$ in the presence of DMEE (5.3 $\mathrm{mg} ; 0.010 \mathrm{mmol})$ in DMF $(1.0 \mathrm{~mL})$ was performed for 6 hours under the irradiation of $365 \mathrm{~nm}$. The resulting solution was precipitated into an excess amount of methanol to obtain the corresponding polymer 12 as a pale yellow solid (248 mg; $0.480 \mathrm{mmol} ; 96 \%) ;{ }^{1} \mathrm{H}-\mathrm{NMR}(400 \mathrm{MHz}$, DMSO- $d_{6}$, at r.t., ppm) $\delta 6.36$ (brs, $\left.1 \mathrm{H}\right), 5.64$ (brs, $2 \mathrm{H}$ ), 4.11-3.91 (brm, 8H), 3.36 (brs, 2H), 3.08 (brs, 4H), 2.52-2.28 (brs, $1 \mathrm{H}), 1.90-1.25$ (brm, $18 \mathrm{H}), 0.90-0.84$ (brm, $9 \mathrm{H}) ;{ }^{13} \mathrm{C}-$ NMR (100 MHz, $\mathrm{CDCl}_{3}$, at r.t., ppm) $\delta 174.55,157.09,156.76,66.55,66.19,64.13,63.73$, 41.90, 41.17, 39.98, 38.90, 29.93, 26.57, 22.64, 16.77, 14.10; IR (ATR, $\left.\mathrm{cm}^{-1}\right) v 3315$, 2941, 2926, 2856, 1693, 1529, 1450, 1414, 1333, 1240, 1147, 1111, 1022, 833, 773, 725.

\section{Radical photopolymerization of acrylate 11}

According to the typical procedure for the synthesis of $\mathbf{7}$, radical photopolymerization of 11 (264 mg; $0.500 \mathrm{mmol})$ in the presence of DMEE (5.3 mg; $0.010 \mathrm{mmol})$ in DMF $(1.0 \mathrm{~mL})$ was performed for 4 hours under the irradiation of $365 \mathrm{~nm}$. The resulting 
solution was precipitated into an excess amount of methanol to obtain the corresponding polymer 13 as a pale yellow solid (244 mg; $0.462 \mathrm{mmol} ; 92 \%) ;{ }^{1} \mathrm{H}-\mathrm{NMR}$ (400 MHz, DMSO- $d_{6}$, at r.t., ppm) $\delta 6.99$ (brs, 2H), 5.02-4.56 (brm, 3H), 4.09-3.96 (brm, 2H), 3.18 (brs, 2H), 2.93 (brs, 4H), 2.19-1.94 (brm, 4H), 1.54-1.21 (brm, 21H), 0.83 (brs, 6H); ${ }^{13} \mathrm{C}-$ $\mathrm{NMR}\left(100 \mathrm{MHz}, \mathrm{CDCl}_{3}\right.$, at r.t., ppm) $\delta 174.63,155.99,155.81,68.84,67.82,67.34,64.05$, 63.59, 41.74, 41.07, 40.04, 37.45, 35.57, 31.56, 29.96, 26.54, 22.64, 14.11; IR (ATR, cm ${ }^{-}$ $\left.{ }^{1}\right) v 3317,2945,2927,2858,1693,1514,1456,1377,1298,1228,1130,1034,1009,773$, 725. 
S2. NMR data

${ }^{1} \mathrm{H}-\mathrm{NMR}$
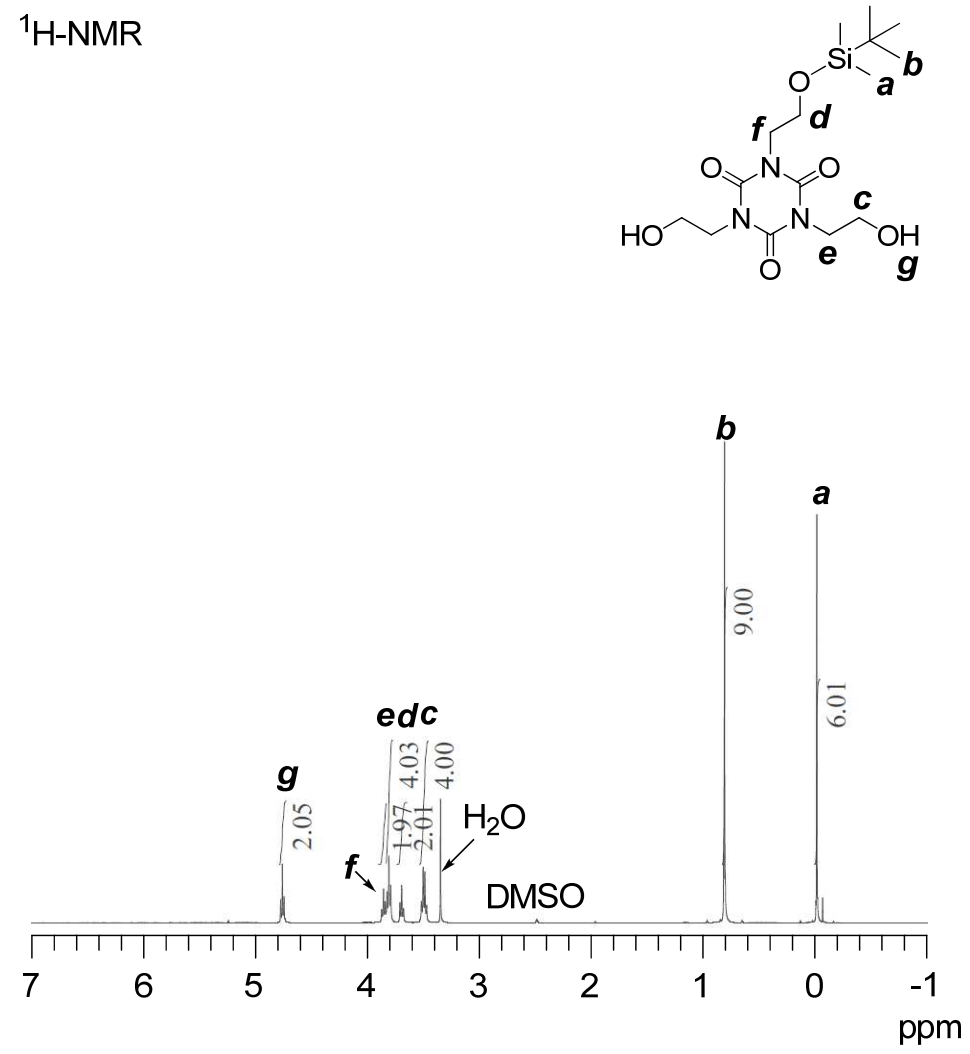

${ }^{13} \mathrm{C}-\mathrm{NMR}$

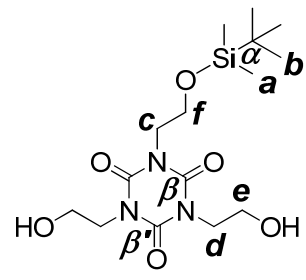

DMSO

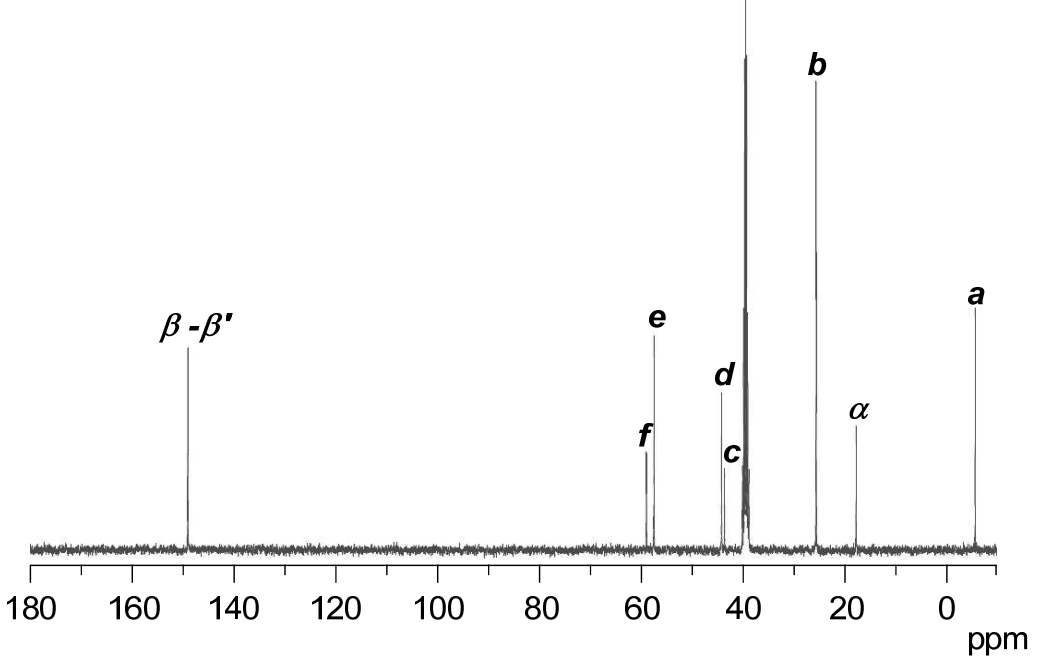

Figure S1. ${ }^{1} \mathrm{H}$ - and ${ }^{13} \mathrm{C}$-NMR spectra of diol $\mathbf{1}$ 
${ }^{1} \mathrm{H}-{ }^{13} \mathrm{C}-\mathrm{NMR}$
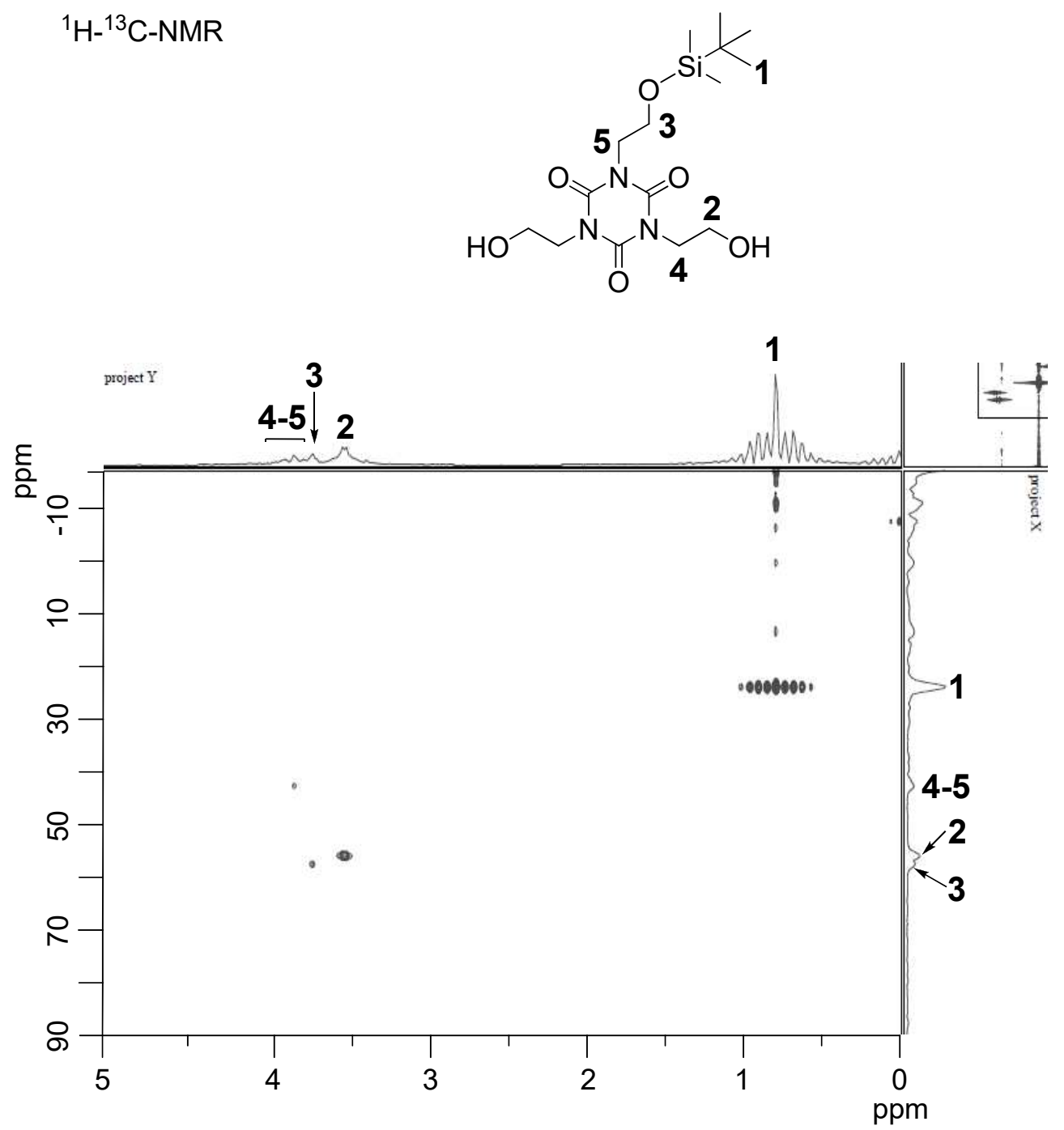

Figure S2. ${ }^{1} \mathrm{H}-{ }^{13} \mathrm{C}-\mathrm{NMR}$ spectrum of diol $\mathbf{1}$ 
${ }^{1} \mathrm{H}-\mathrm{NMR}$
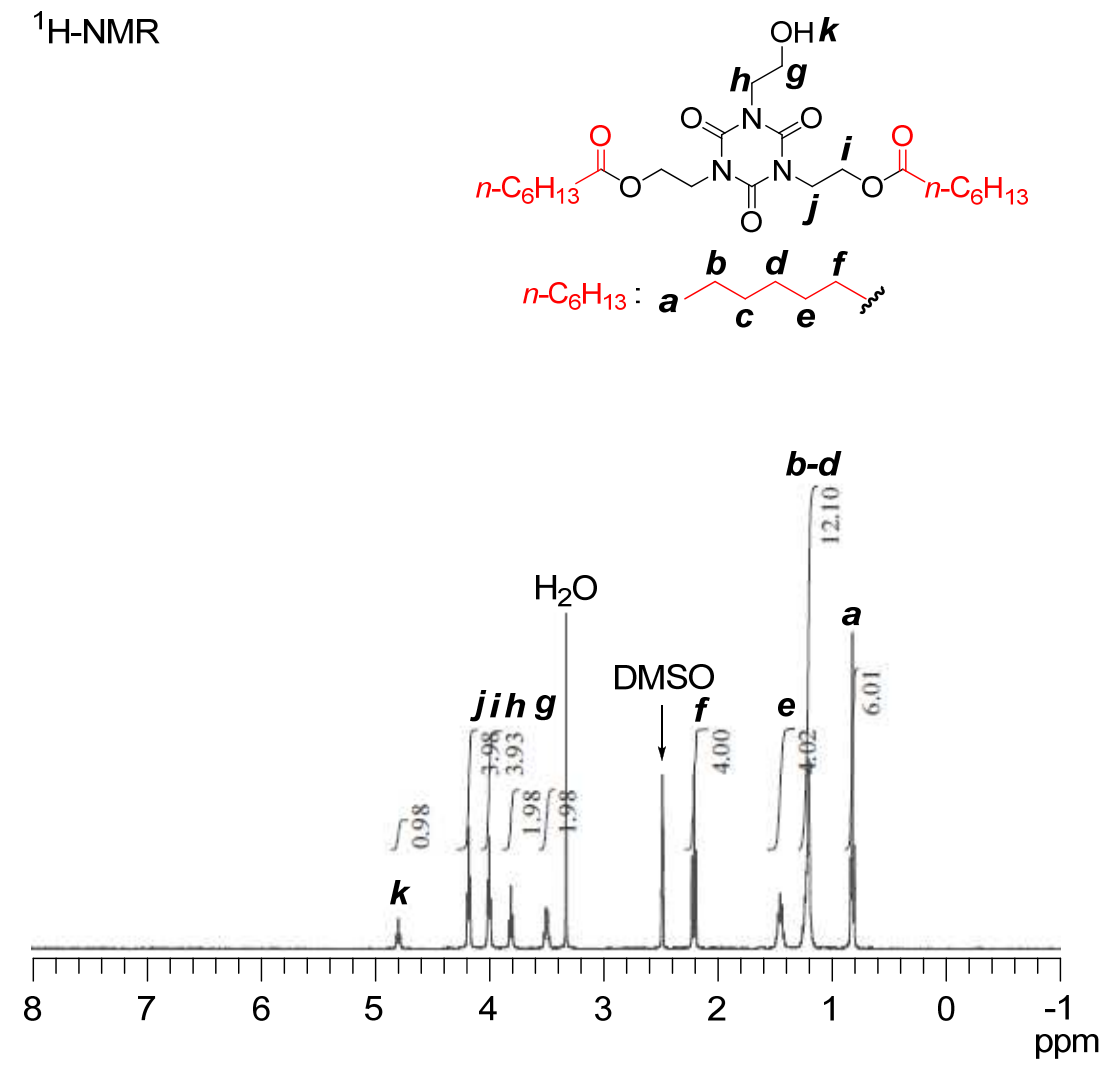

${ }^{13} \mathrm{C}-\mathrm{NMR}$
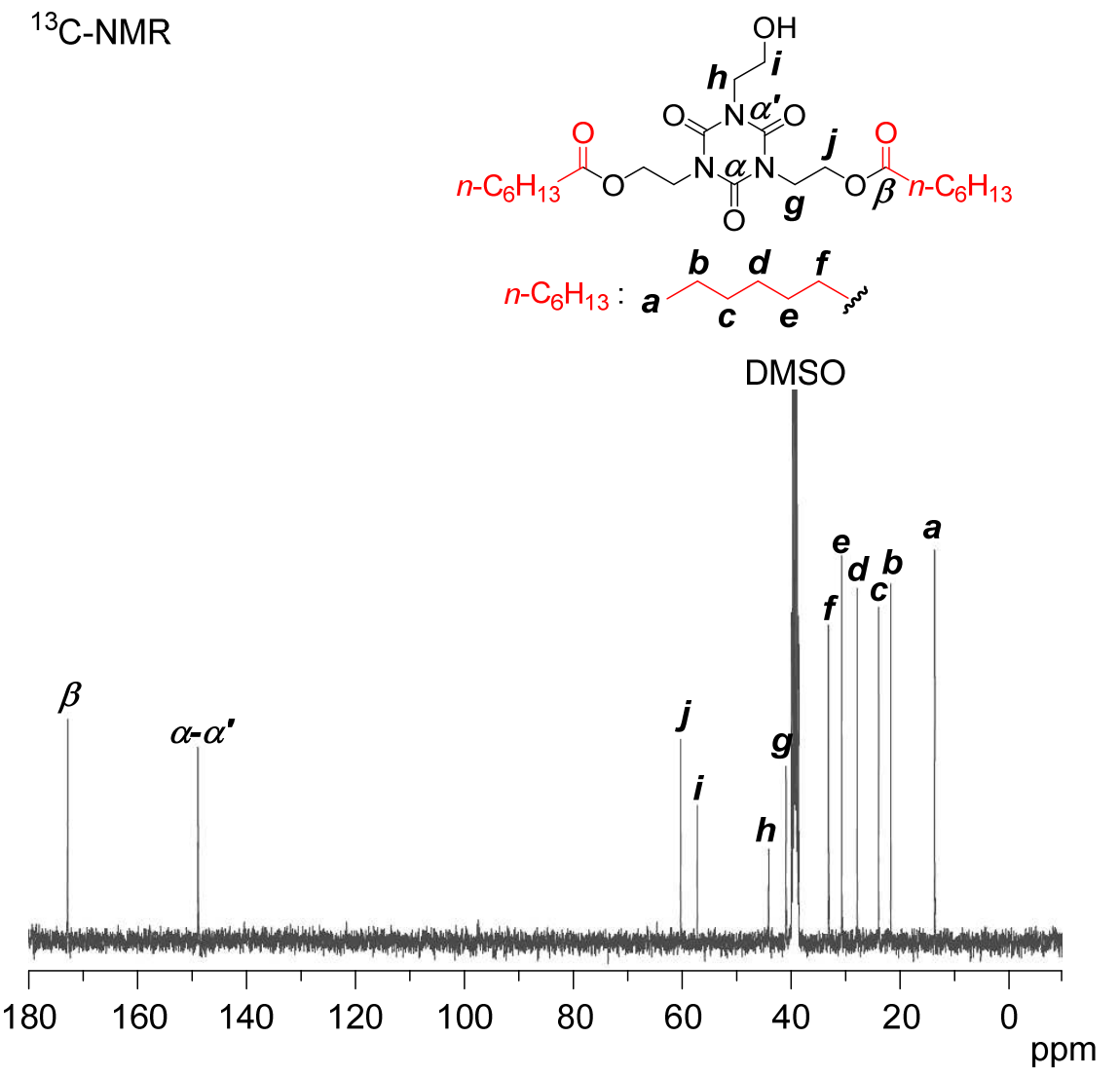

Figure S3. ${ }^{1} \mathrm{H}$ - and ${ }^{13} \mathrm{C}-\mathrm{NMR}$ spectra of monool 2 

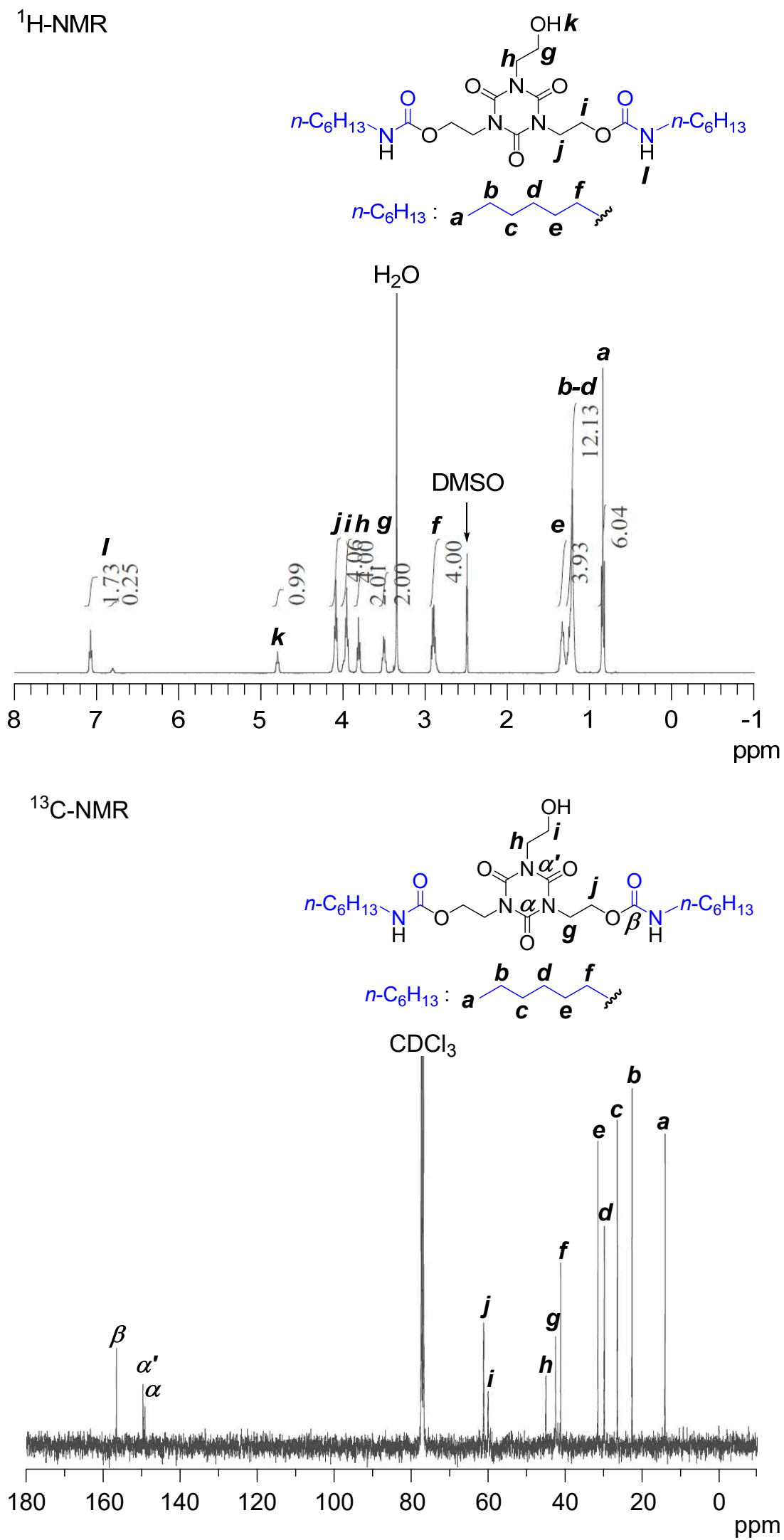

Figure $\mathrm{S} 4 .{ }^{1} \mathrm{H}$ - and ${ }^{13} \mathrm{C}-\mathrm{NMR}$ spectra of monool 3 


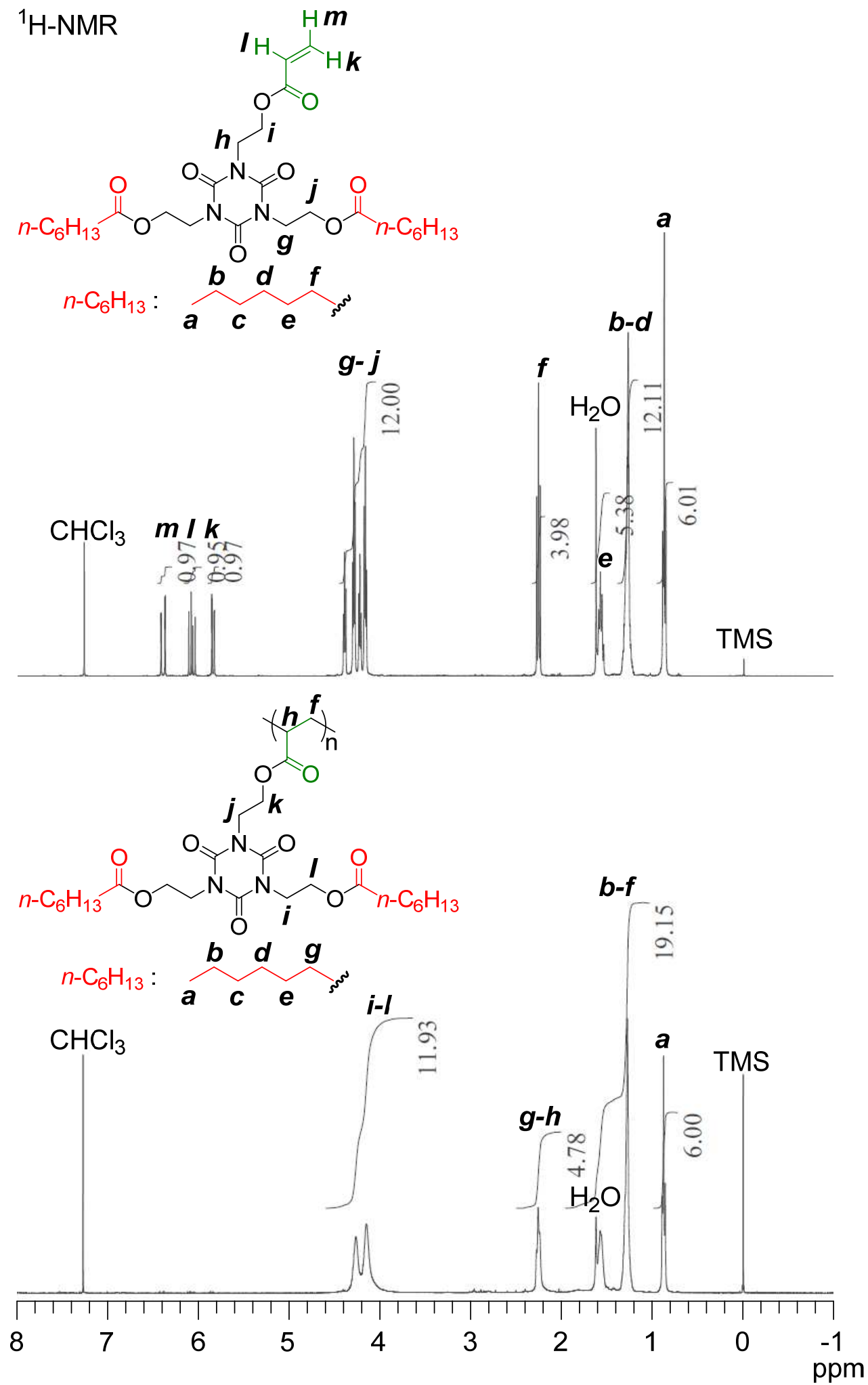

Figure S5. ${ }^{1} \mathrm{H}-\mathrm{NMR}$ spectra of acrylate $\mathbf{4}$ and polymer $\mathbf{7}$ 


$$
{ }^{13} \mathrm{C}-\mathrm{NMR}
$$

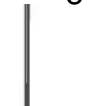

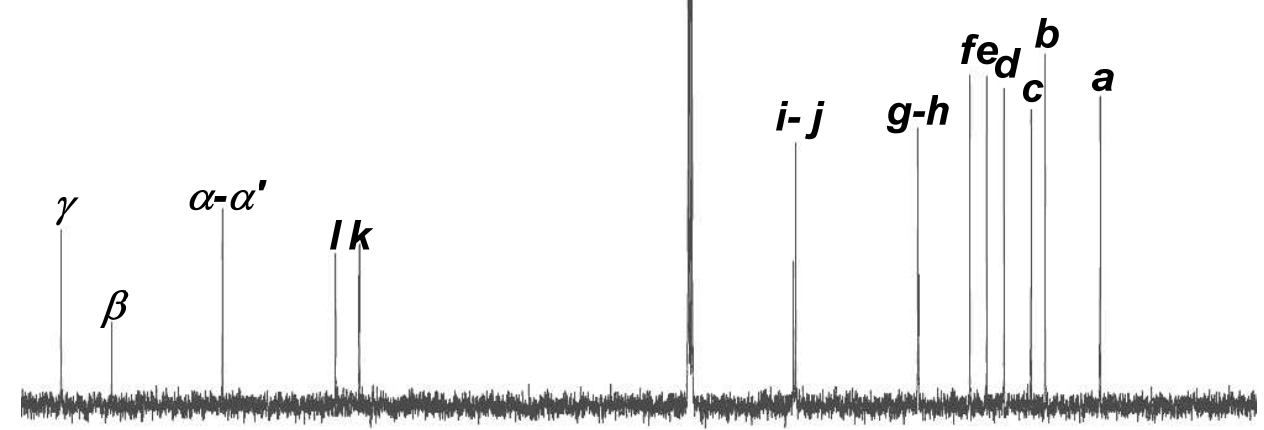

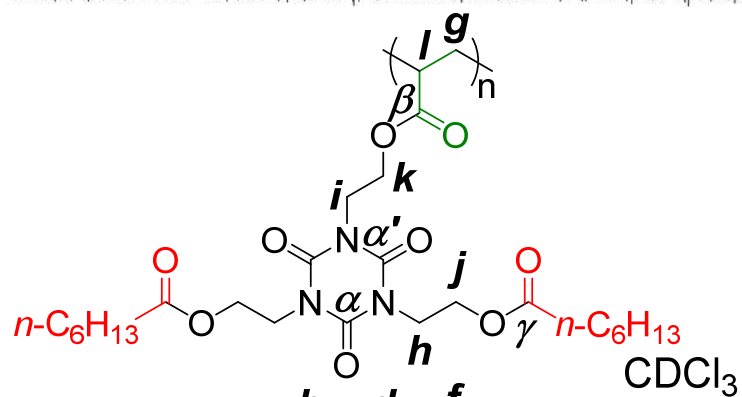

$$
n-\mathrm{C}_{6} \mathrm{H}_{13}: \overbrace{\boldsymbol{a}}^{\boldsymbol{b}} \boldsymbol{d} \boldsymbol{f} \mathrm{s}^{\mathrm{s}}
$$

$\mathrm{CDCl}_{3}$
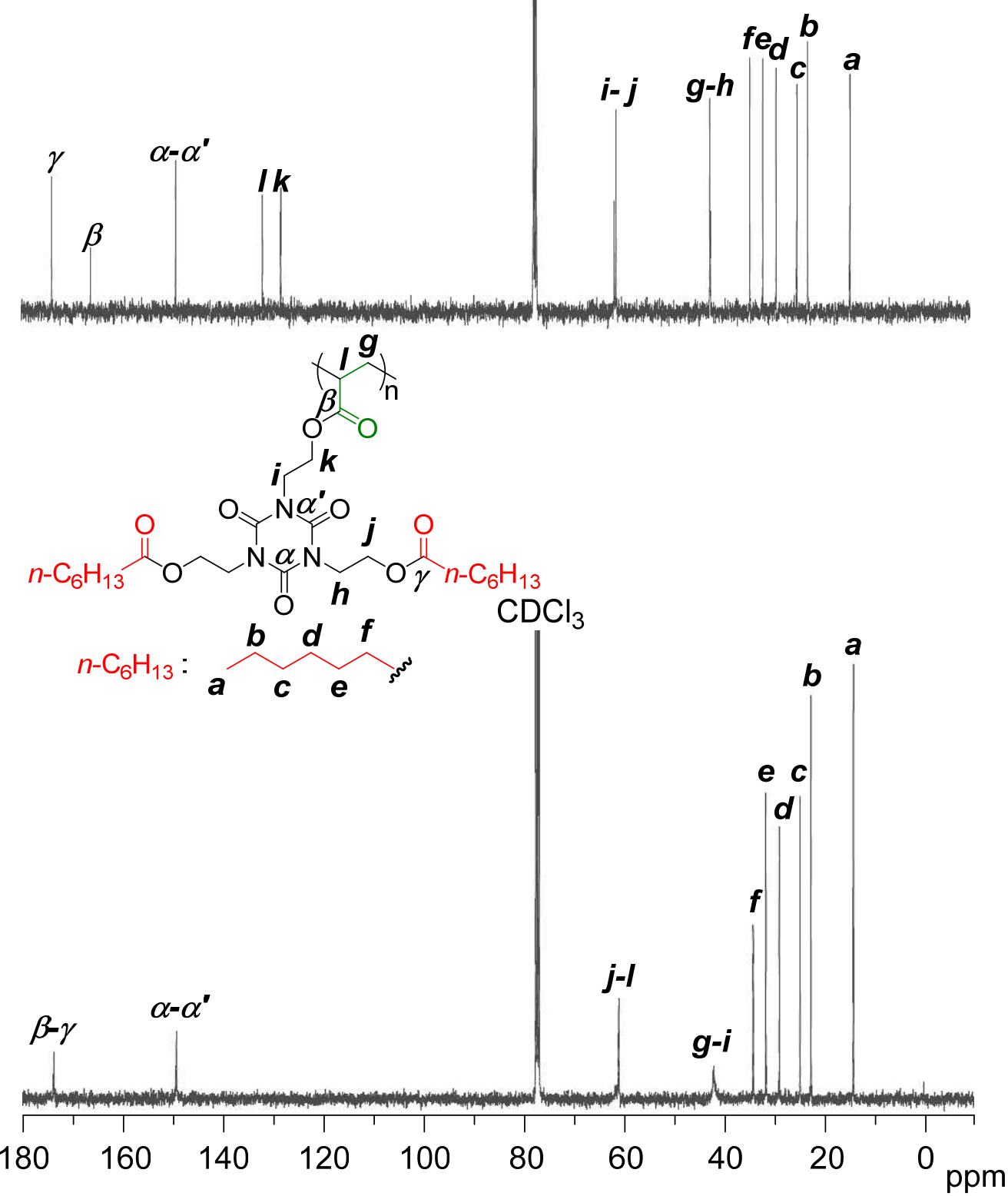

Figure S6. ${ }^{13} \mathrm{C}-\mathrm{NMR}$ spectra of acrylate $\mathbf{4}$ and polymer $\mathbf{7}$ 

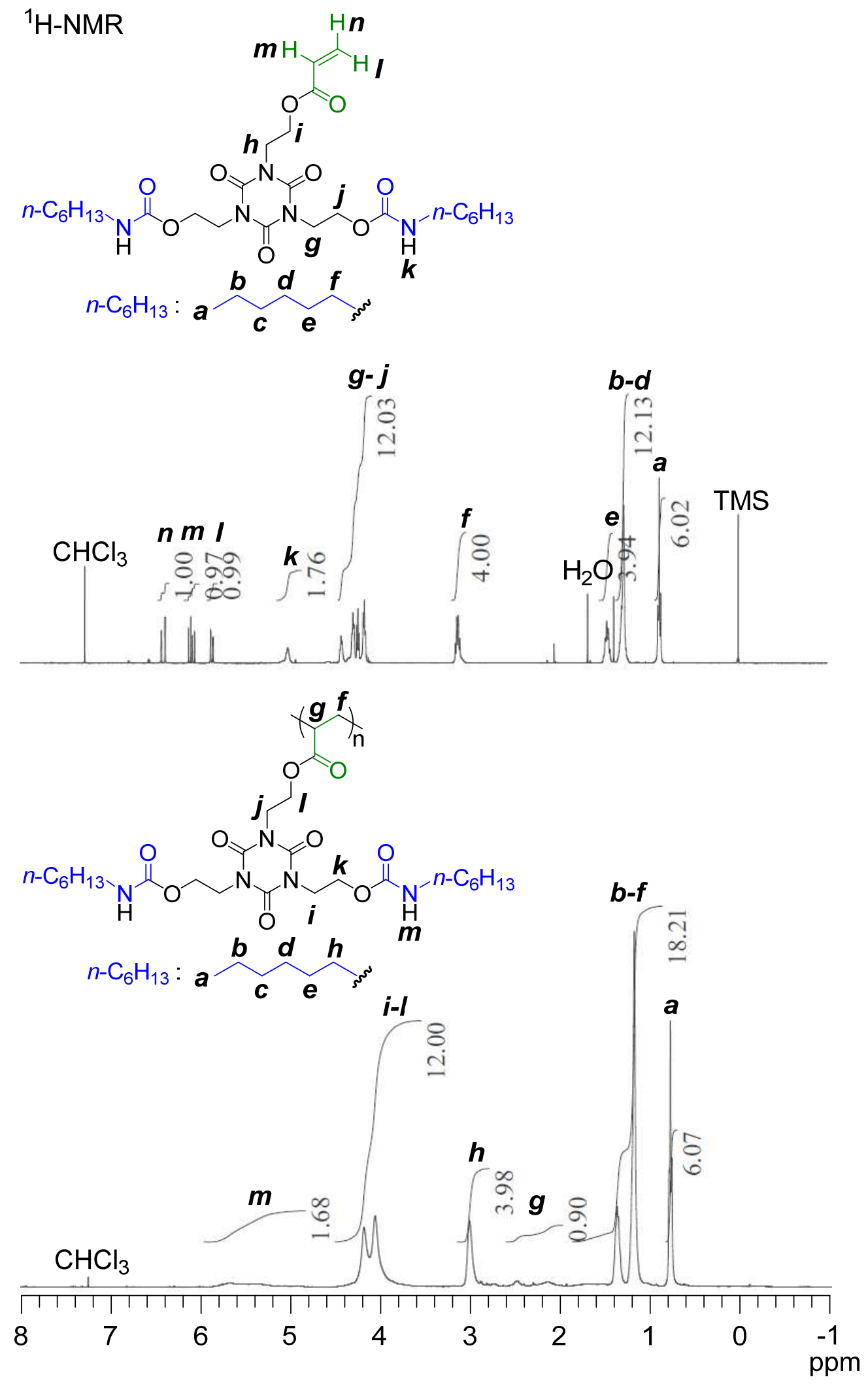

Figure S7. ${ }^{1} \mathrm{H}-\mathrm{NMR}$ spectra of acrylate $\mathbf{5}$ and polymer $\mathbf{8}$ 
${ }^{13} \mathrm{C}-\mathrm{NMR}$

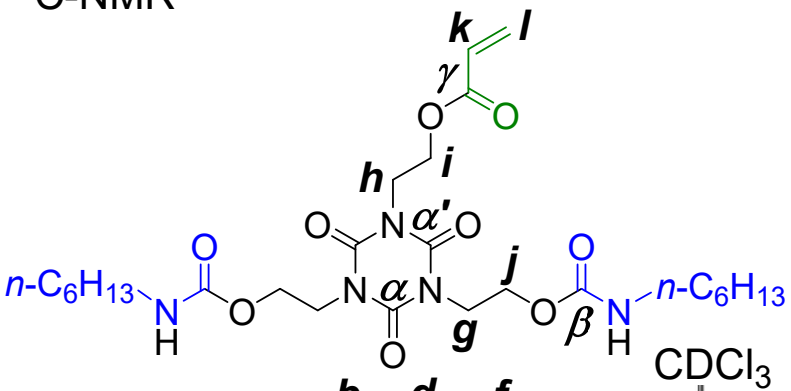

$$
\begin{aligned}
& n-\mathrm{C}_{6} \mathrm{H}_{13}: \overbrace{\boldsymbol{c}}^{\boldsymbol{b}} \overbrace{\boldsymbol{e}}^{\boldsymbol{d}} \boldsymbol{f}
\end{aligned}
$$

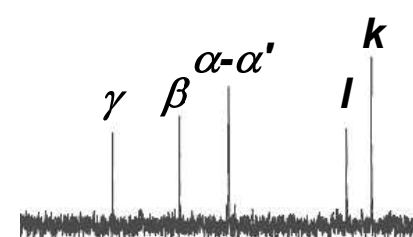

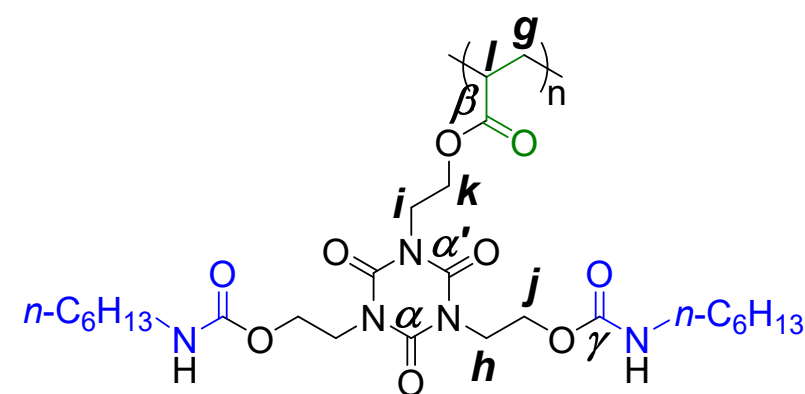

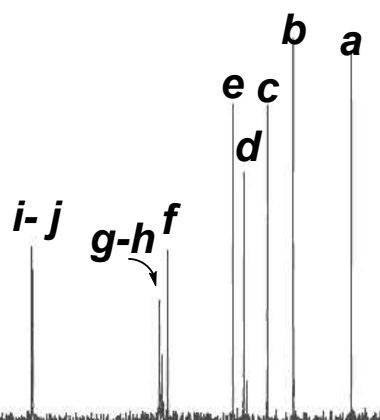

$n-\mathrm{C}_{6} \mathrm{H}_{13}: \boldsymbol{a} \overbrace{\boldsymbol{c}}^{\boldsymbol{b}} \boldsymbol{d} \boldsymbol{f}$

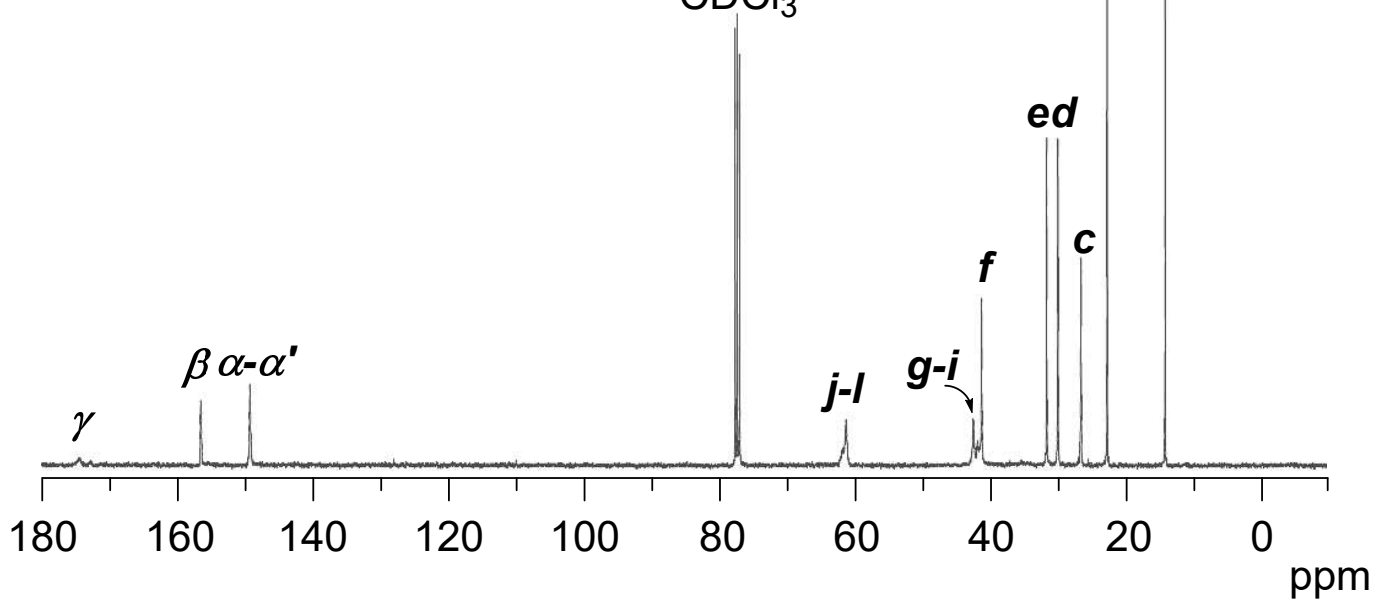

Figure S8. ${ }^{13} \mathrm{C}$-NMR spectra of acrylate 5 and polymer $\mathbf{8}$ 


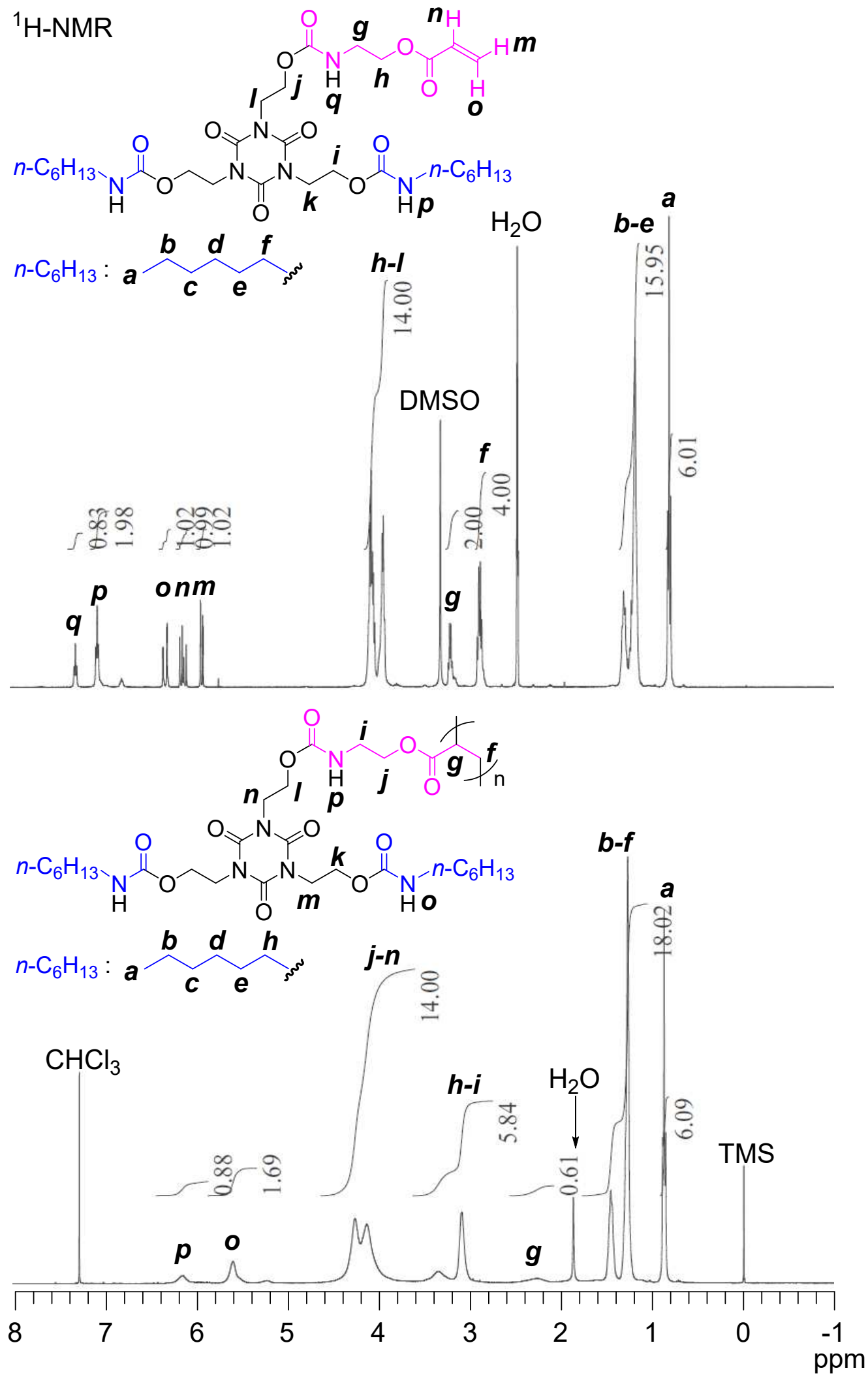

Figure S9. ${ }^{1} \mathrm{H}-\mathrm{NMR}$ spectra of acrylate 6 and polymer 9 


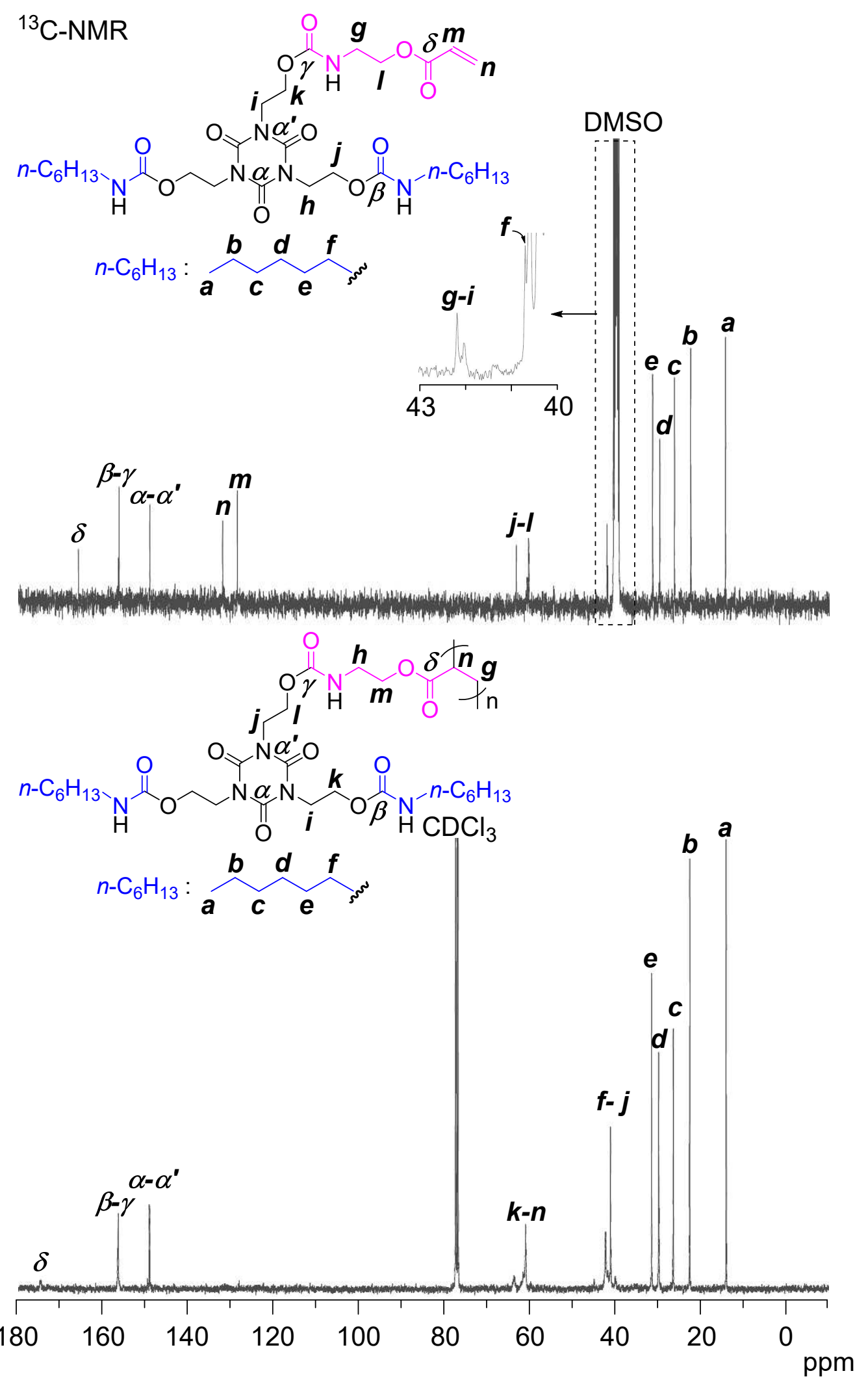

Figure S10. ${ }^{13} \mathrm{C}-\mathrm{NMR}$ spectra of acrylate $\mathbf{6}$ and polymer $\mathbf{9}$ 
${ }^{1} \mathrm{H}-{ }^{13} \mathrm{C}-\mathrm{NMR}$

$$
{ }_{n-\mathrm{C}_{6} \mathrm{H}_{13}}: \underbrace{2}_{\mathbf{3}}
$$

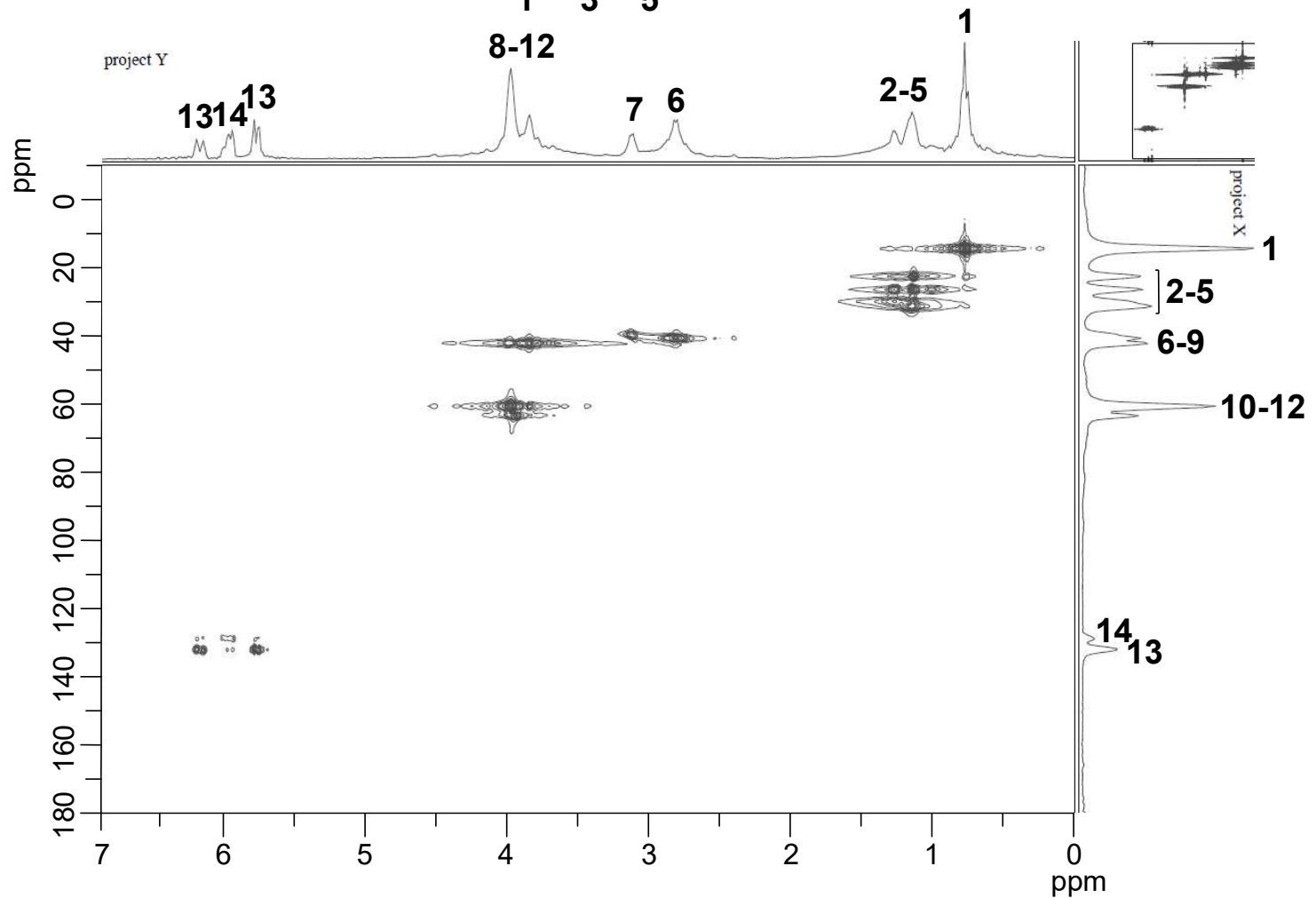

Figure S11. ${ }^{1} \mathrm{H}^{-13} \mathrm{C}-\mathrm{NMR}$ spectrum of acrylate 6 


\section{${ }^{1} \mathrm{H}-\mathrm{NMR}$}

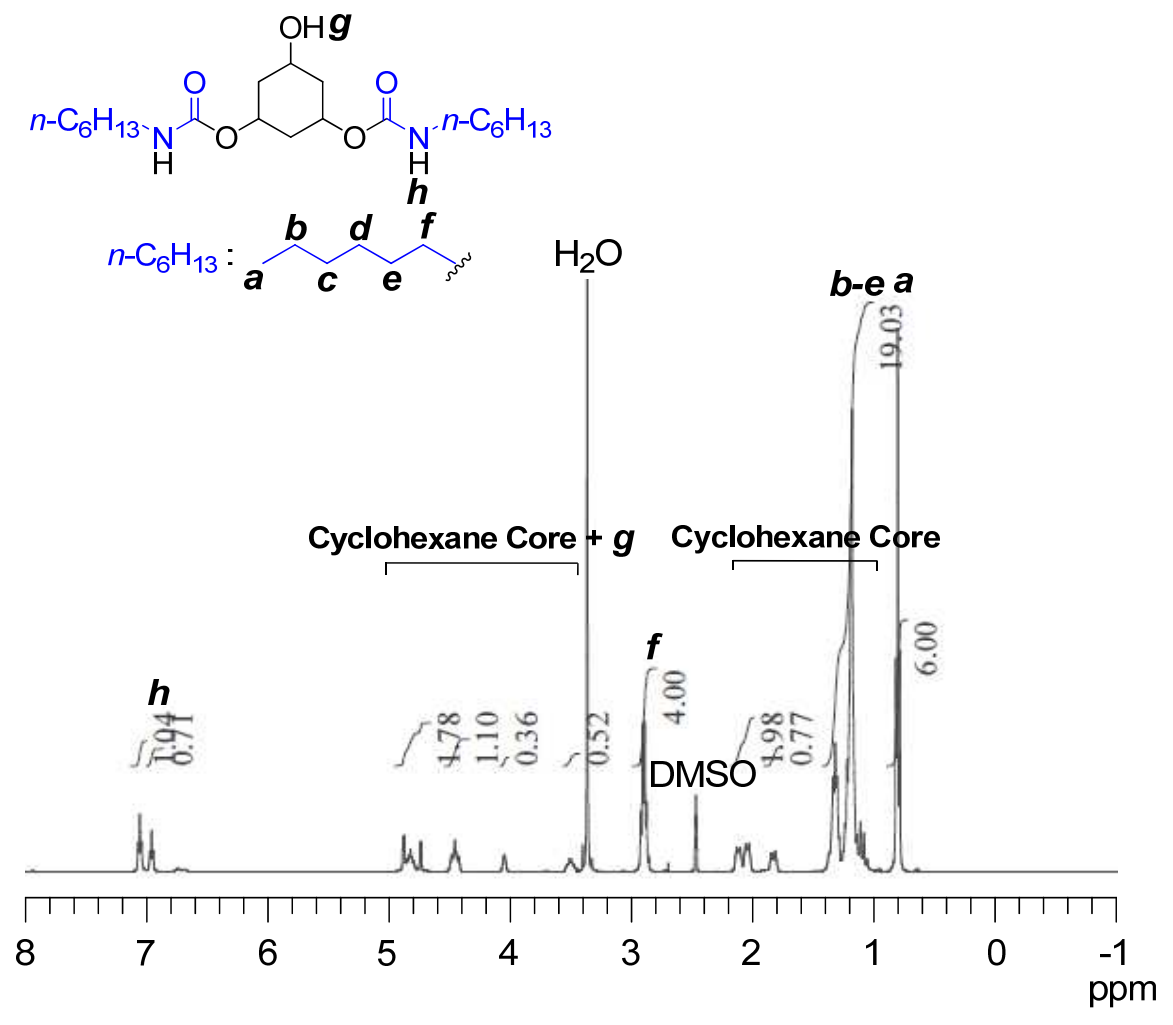

${ }^{13} \mathrm{C}-\mathrm{NMR}$

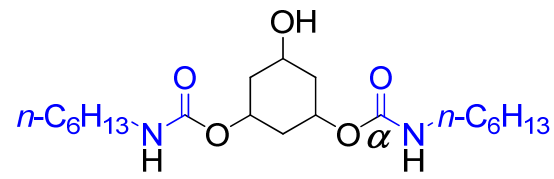

$$
n-\mathrm{C}_{6} \mathrm{H}_{13}: \overbrace{\boldsymbol{a}}^{\boldsymbol{b}} \boldsymbol{d} \underbrace{\boldsymbol{f}}_{\text {e }}
$$
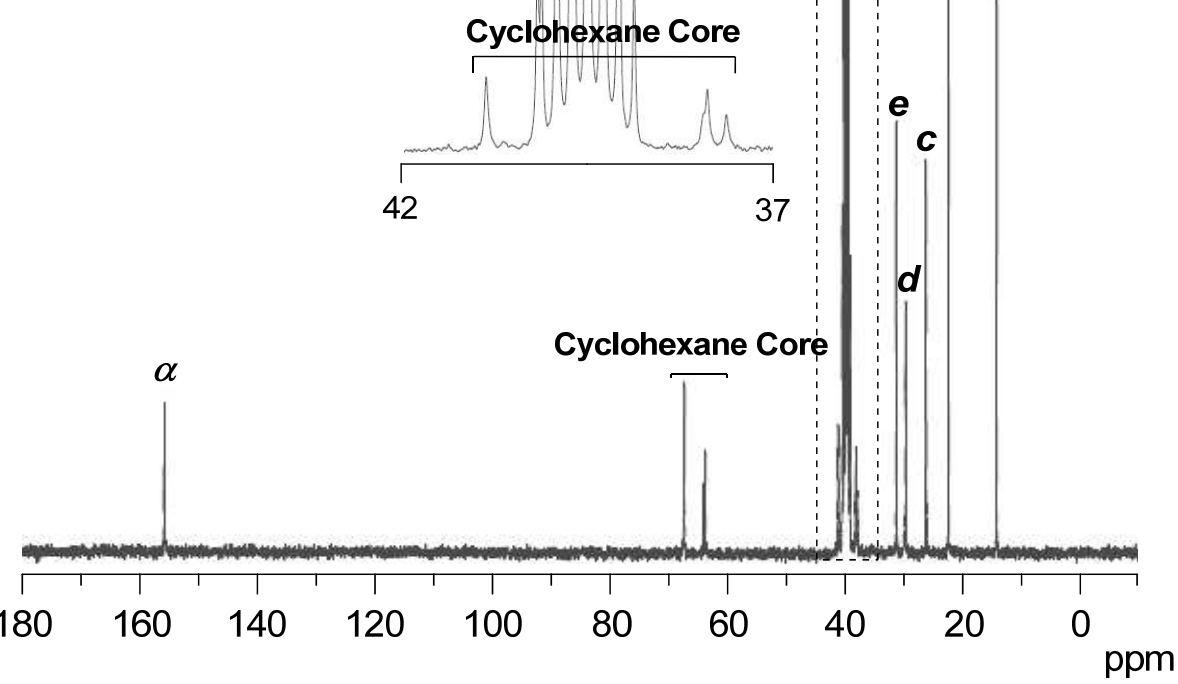

Figure $\mathrm{S} 12 .{ }^{1} \mathrm{H}$ - and ${ }^{13} \mathrm{C}$-NMR spectra of monool bearing a cyclohexane core 


\section{${ }^{1} \mathrm{H}-\mathrm{NMR}$}

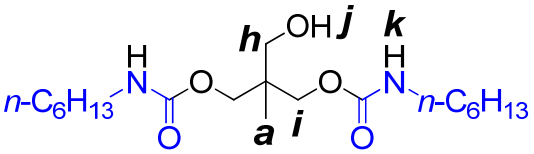

$$
\begin{aligned}
& n-\mathrm{C}_{6} \mathrm{H}_{13}: \overbrace{\boldsymbol{b}}^{\boldsymbol{c}} \boldsymbol{d} \overbrace{\boldsymbol{f}}^{\boldsymbol{g}}
\end{aligned}
$$

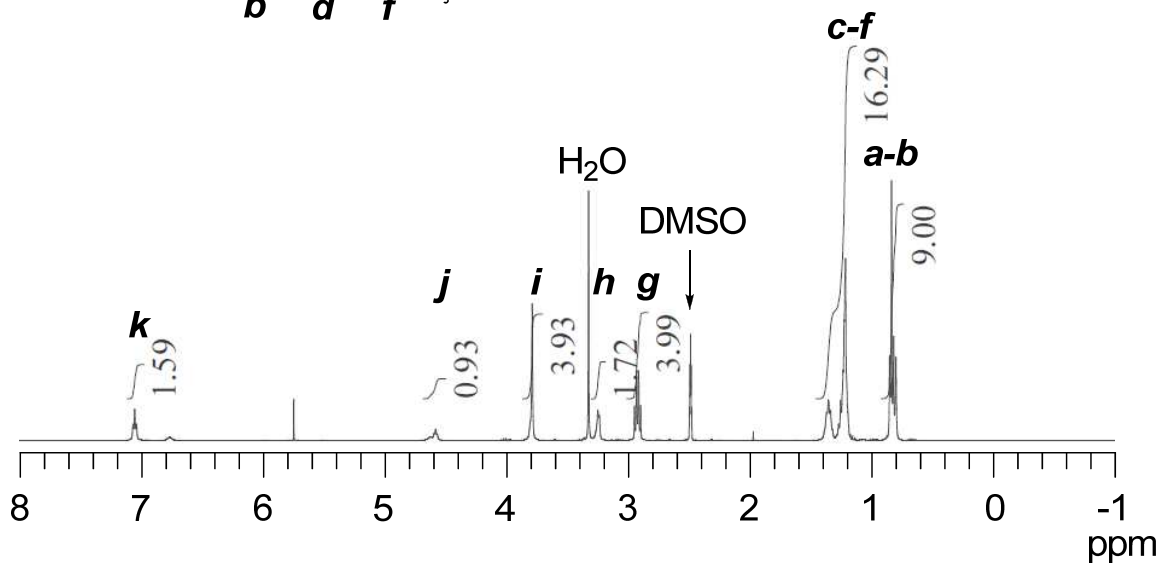

${ }^{13} \mathrm{C}-\mathrm{NMR}$
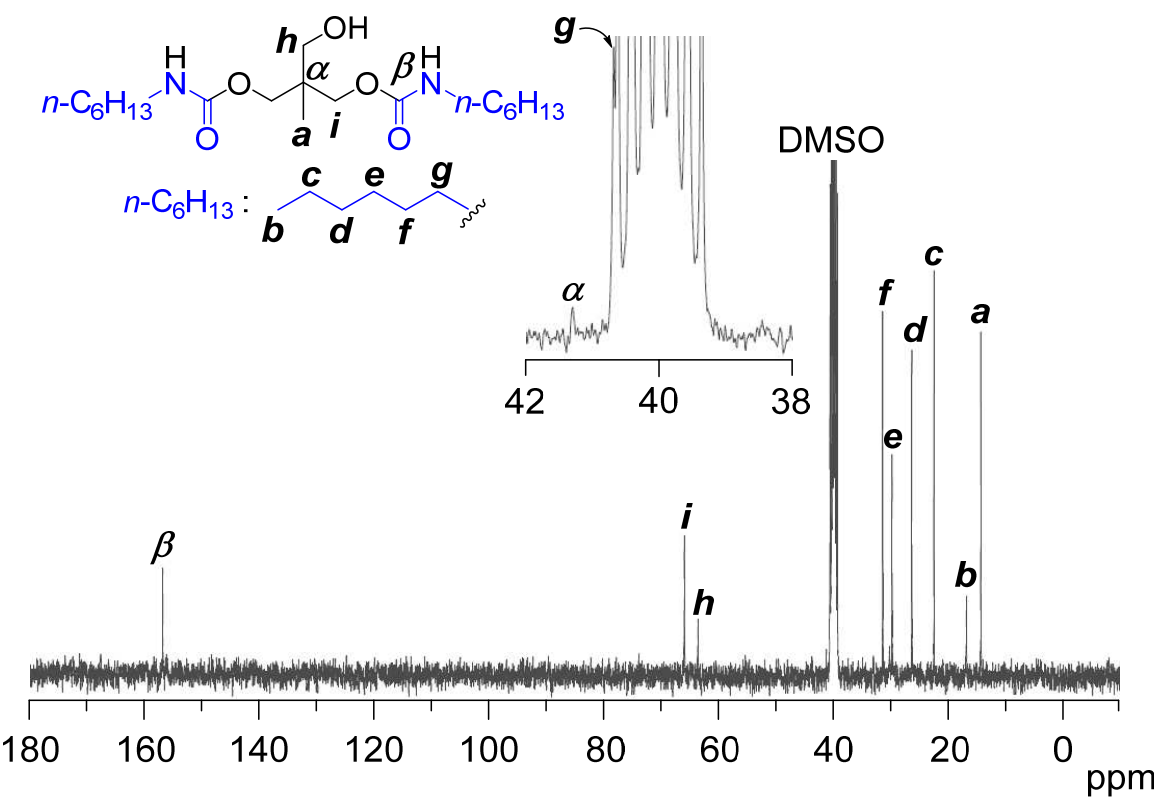

Figure S13. ${ }^{1} \mathrm{H}$ - and ${ }^{13} \mathrm{C}-\mathrm{NMR}$ spectra of monool bearing an alkyl moiety 


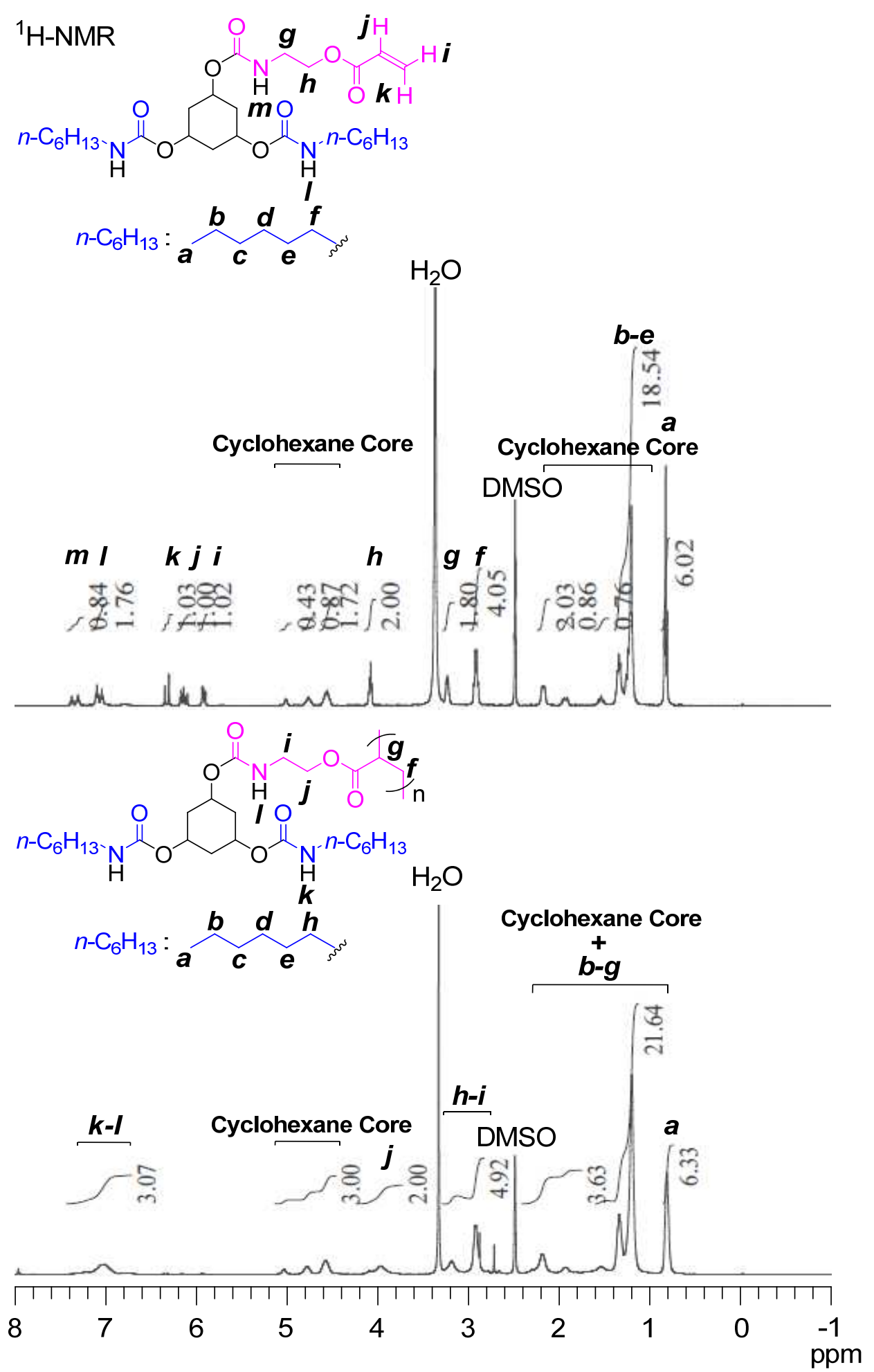

Figure S14. ${ }^{1} \mathrm{H}-\mathrm{NMR}$ spectra of acrylate $\mathbf{1 0}$ and polymer $\mathbf{1 2}$ 


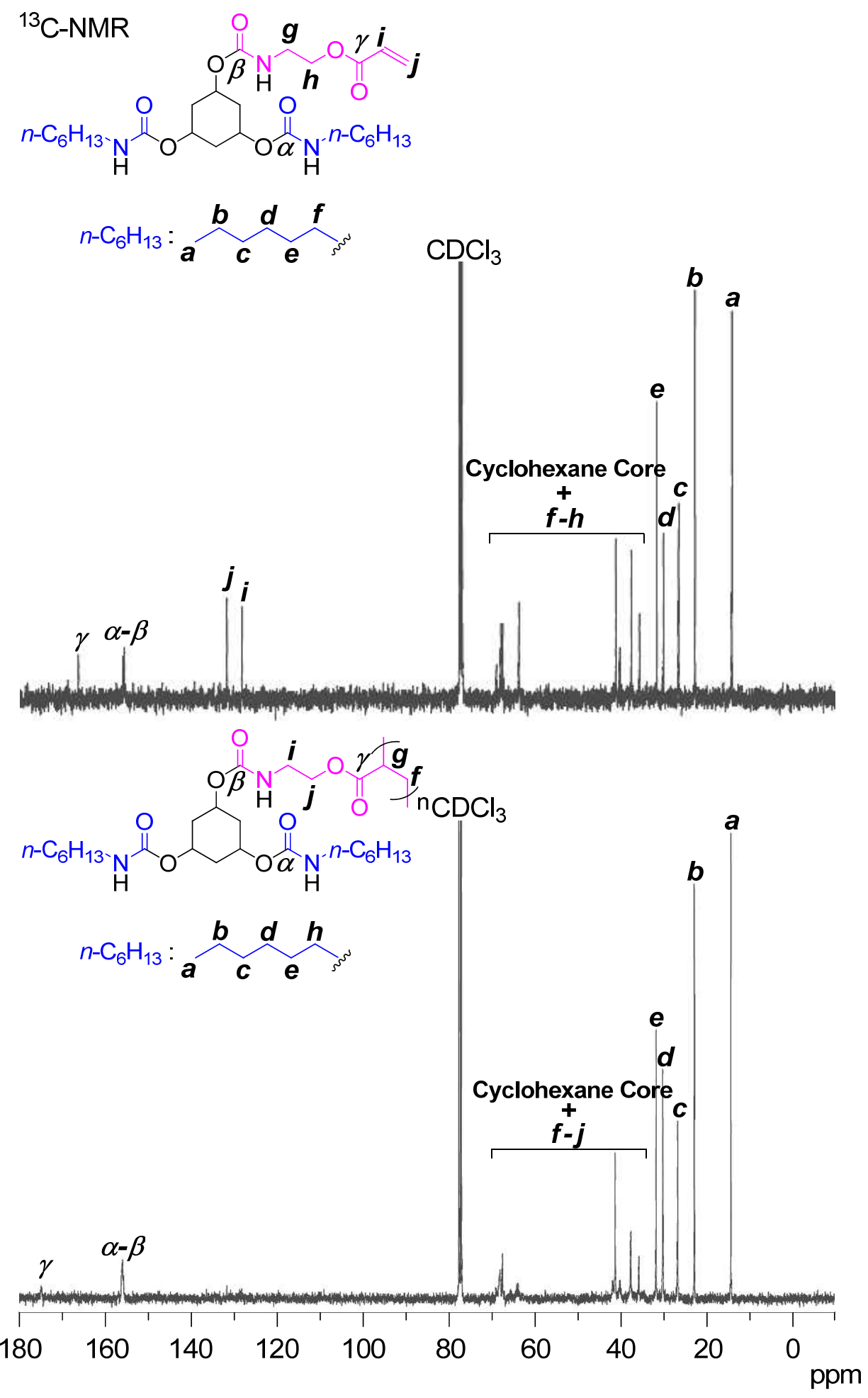

Figure S15. ${ }^{13} \mathrm{C}$-NMR spectra of acrylate $\mathbf{1 0}$ and polymer $\mathbf{1 2}$ 
${ }^{1} \mathrm{H}-\mathrm{NMR}$

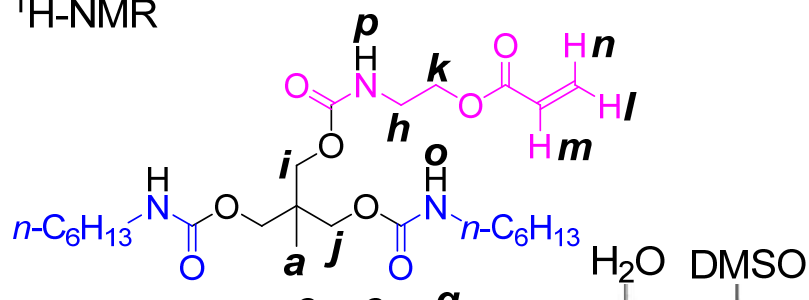
$n-\mathrm{C}_{6} \mathrm{H}_{13}: \overbrace{\boldsymbol{b} \boldsymbol{d}_{\boldsymbol{f}}^{\boldsymbol{c}} \boldsymbol{e}}^{\boldsymbol{g}}$

$\mathrm{H}_{2} \mathrm{O}$ DMSO

po $n$ m l

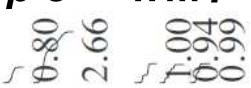

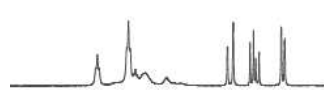

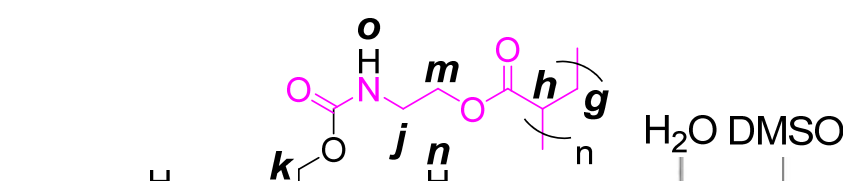

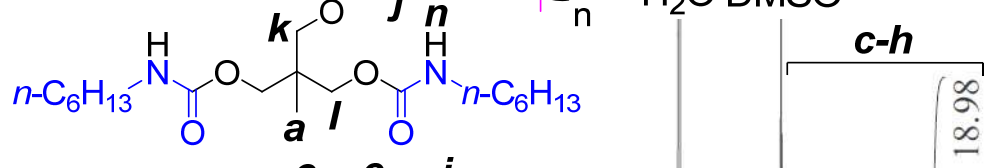
$n-\mathrm{C}_{6} \mathrm{H}_{13}: \overbrace{\boldsymbol{b} \boldsymbol{d}_{\boldsymbol{f}}^{\boldsymbol{c}} \boldsymbol{e} \overbrace{}^{\mathrm{s}}}^{\boldsymbol{i}}$

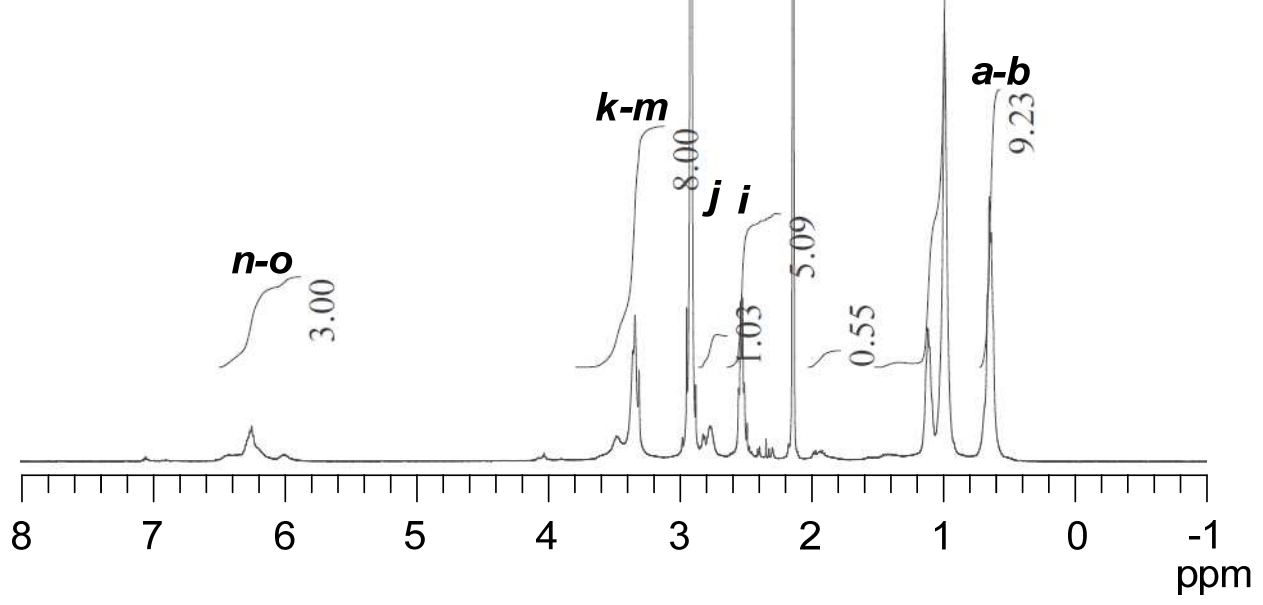

Figure S16. ${ }^{1} \mathrm{H}-\mathrm{NMR}$ spectra of acrylate $\mathbf{1 1}$ and polymer $\mathbf{1 3}$ 


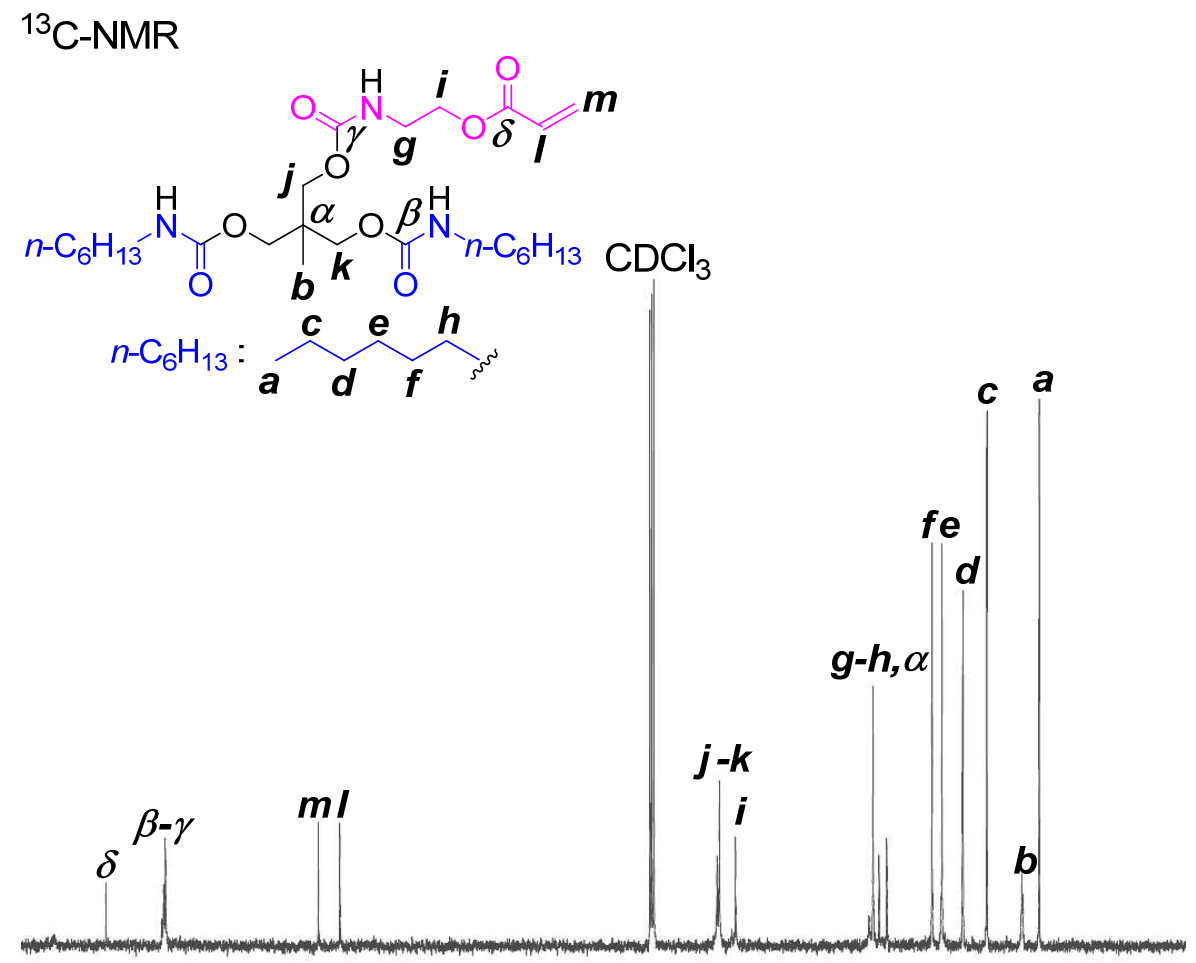

${ }_{1 \gamma}^{\mathrm{N}} \stackrel{\boldsymbol{k}}{\mathrm{H}} \mathrm{\delta} \underbrace{\boldsymbol{j}}_{\mathrm{n}} \mathrm{CDCl}_{3}$

${ }_{n-\mathrm{C}_{6} \mathrm{H}_{13}-\mathrm{N}_{\mathrm{O}}^{\mathrm{H}} \prod_{\mathbf{a}}^{\mathrm{O}} \underbrace{\alpha}_{\boldsymbol{m}} \mathrm{O}}^{\alpha} \prod_{\mathrm{O}}^{\beta} \mathrm{H}_{n-\mathrm{C}_{6} \mathrm{H}_{13}}$

$n-\mathrm{C}_{6} \mathrm{H}_{13}: \overbrace{\boldsymbol{b} \boldsymbol{d}_{\boldsymbol{f}}^{\boldsymbol{c}} \boldsymbol{e}}^{\boldsymbol{h}}$

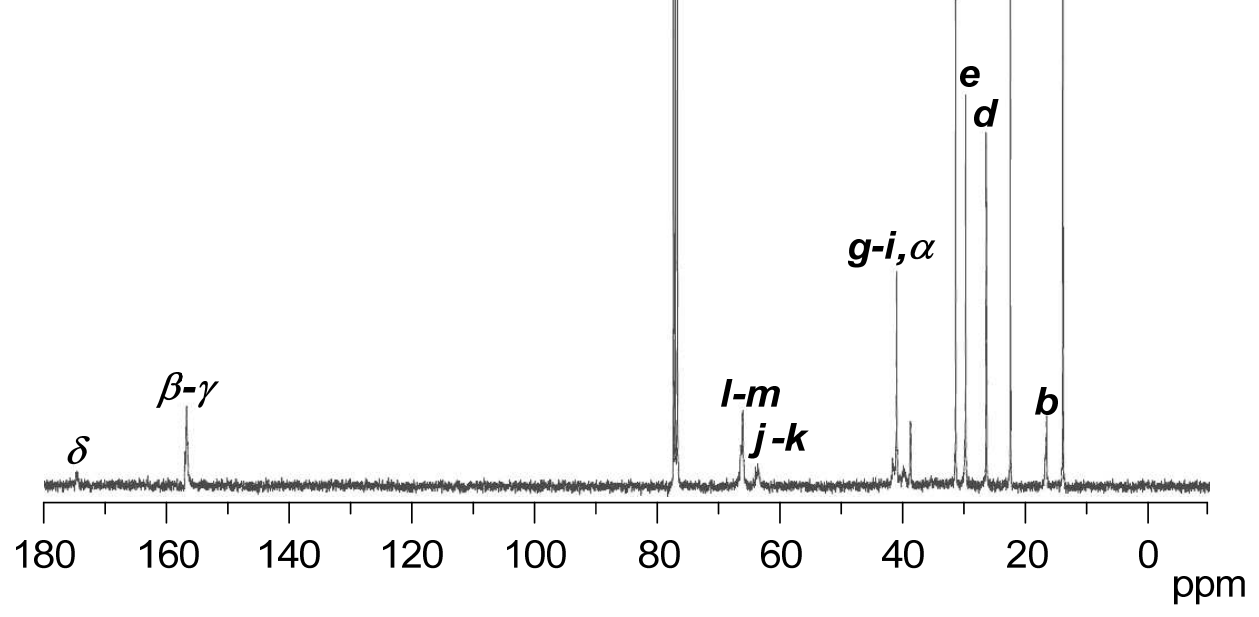

Figure S17. ${ }^{13} \mathrm{C}-\mathrm{NMR}$ spectra of acrylate $\mathbf{1 1}$ and polymer $\mathbf{1 3}$ 
Solid State ${ }^{13} \mathrm{C}-N M R$ (CPMAS)<smiles>CCC(C)C(=O)OCCn1c(=O)n(CCOC(=O)C(C)C)c(=O)n(CC(C)OC(=O)C(C)C)c1=O</smiles>

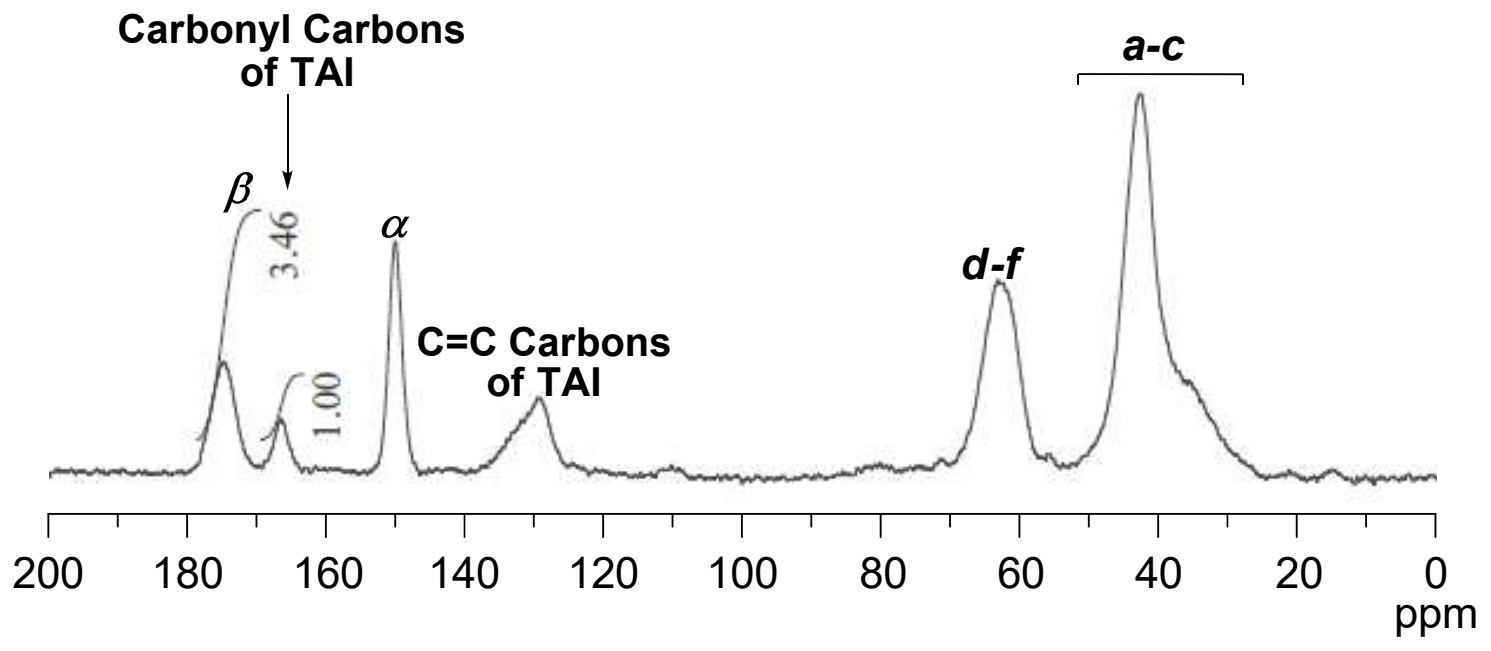

Figure S18. Solid state ${ }^{13} \mathrm{C}-\mathrm{NMR}$ (CPMAS) spectrum of crosslinked polymer 14a 
Solid State ${ }^{13} \mathrm{C}-N M R$ (CPMAS)
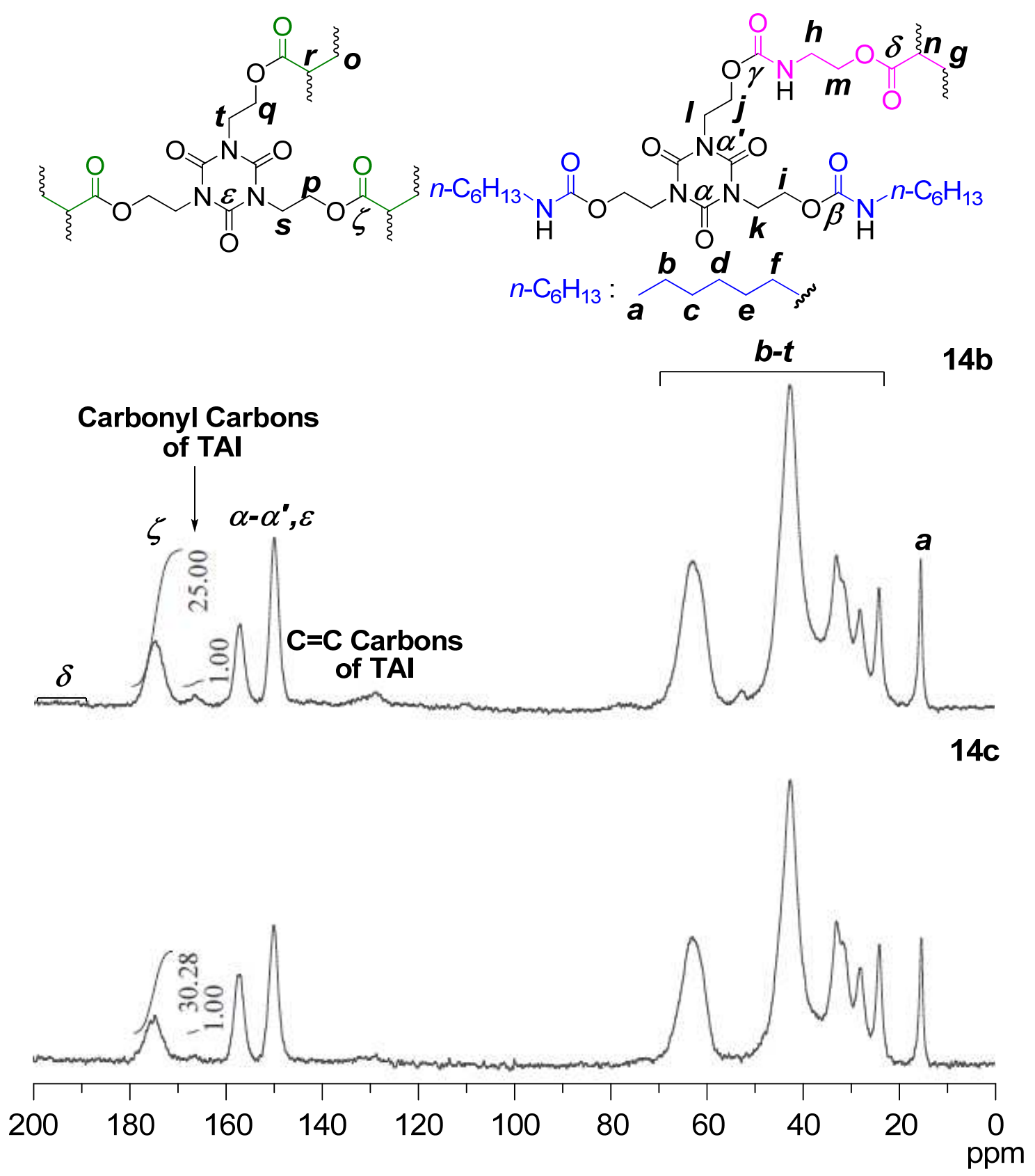

Figure S19. Solid state ${ }^{13}$ C-NMR (CPMAS) spectra of crosslinked polymers $\mathbf{1 4 b - c}$ 


\section{S3. IR data}

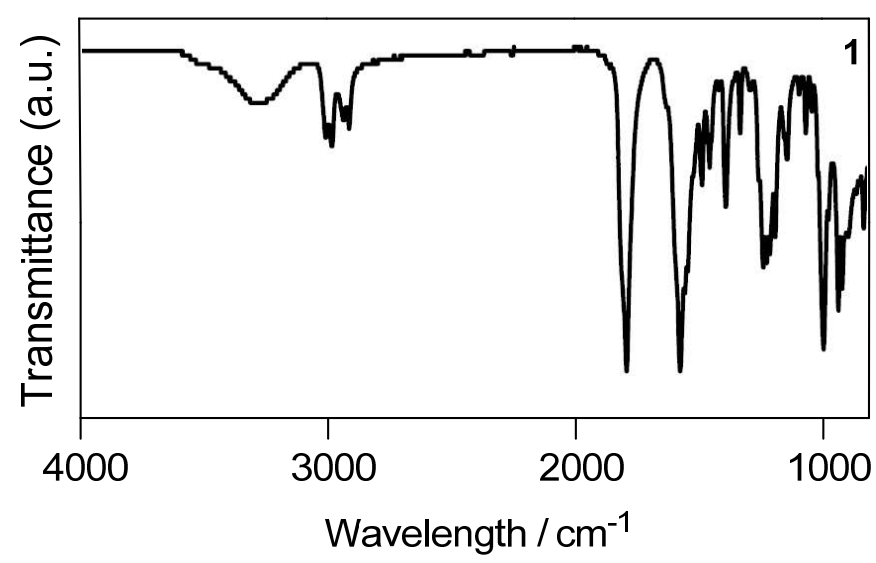

Figure S20. IR spectrum of diol 1

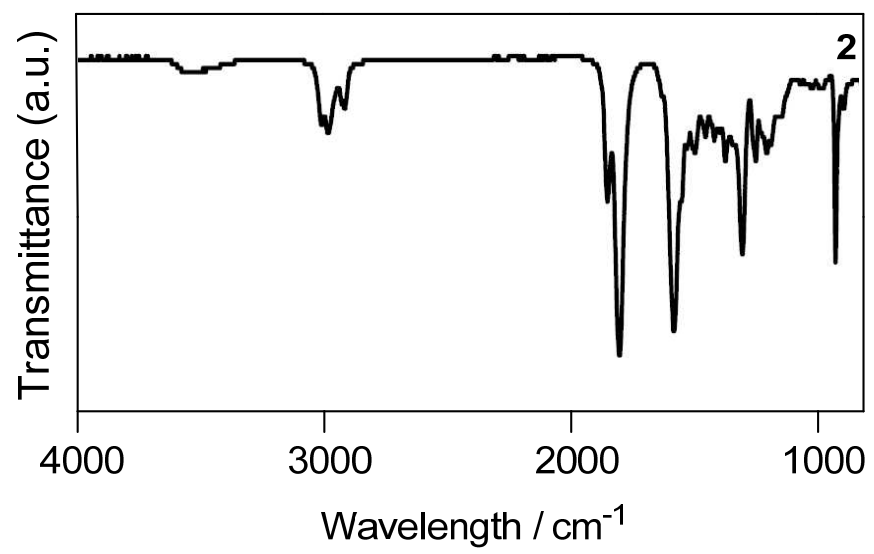

Figure S21. IR spectrum of monool 2

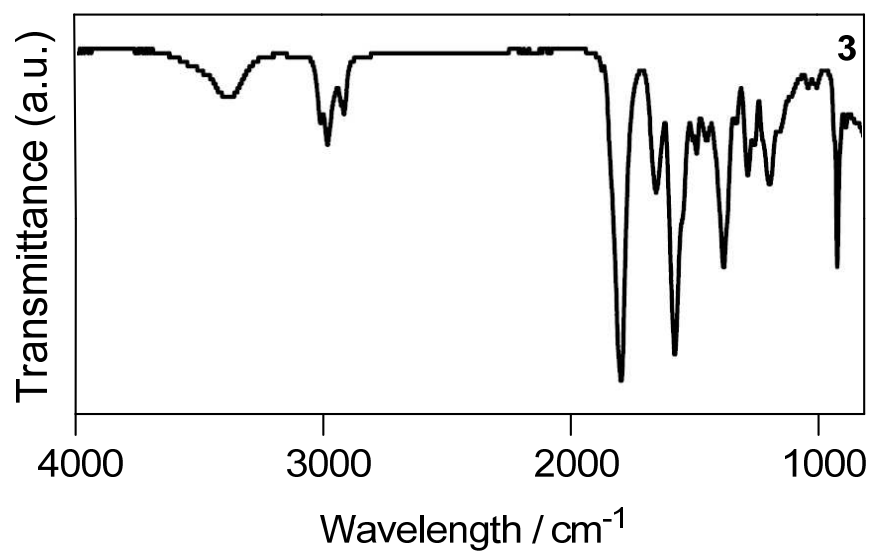

Figure S22. IR spectrum of monool 3 


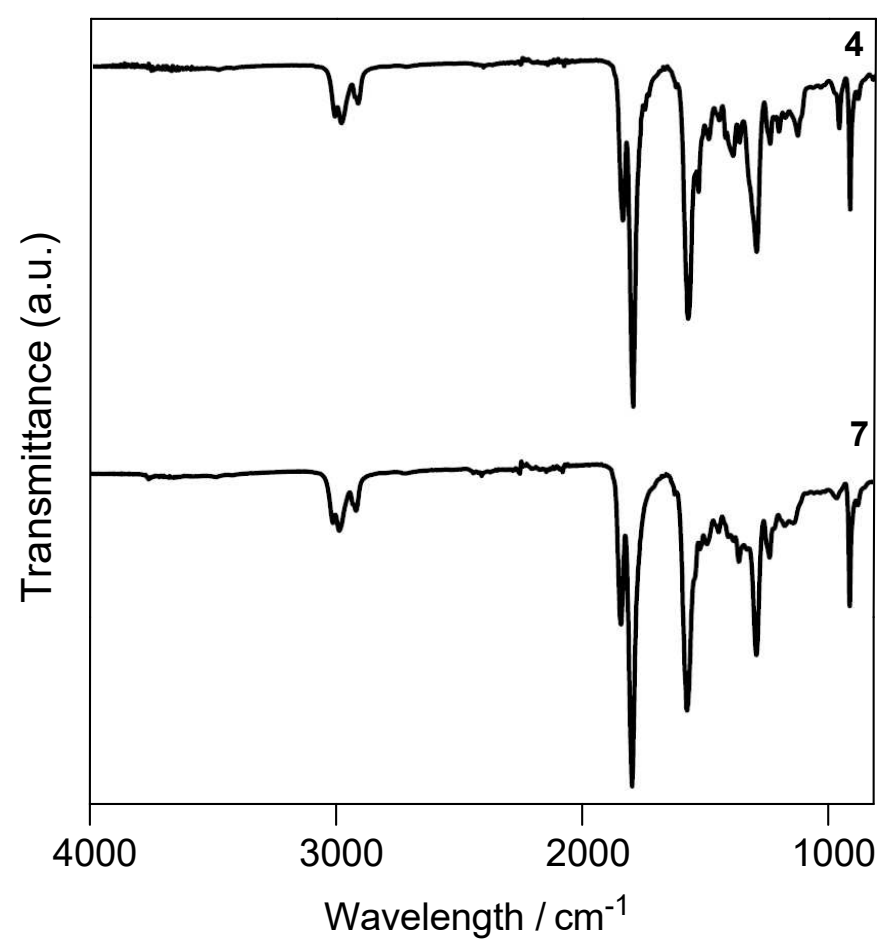

Figure S23. IR spectra of acrylate $\mathbf{4}$ and polymer $\mathbf{7}$

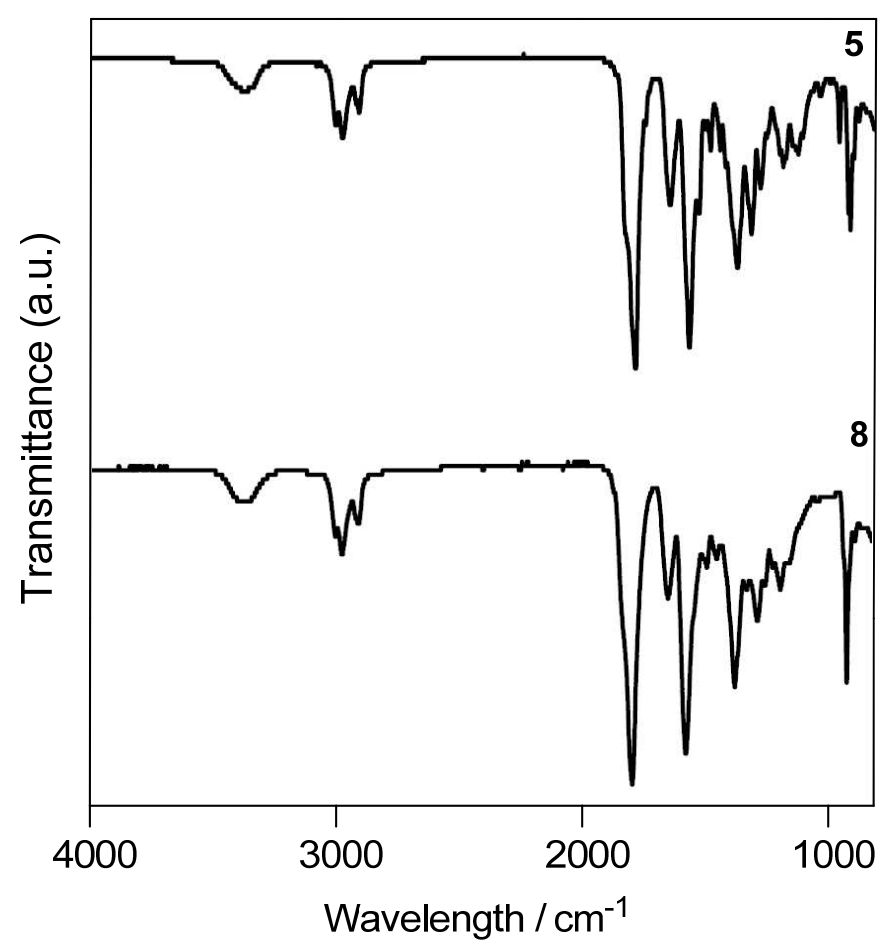

Figure S24. IR spectra of acrylate 5 and polymer $\mathbf{8}$ 


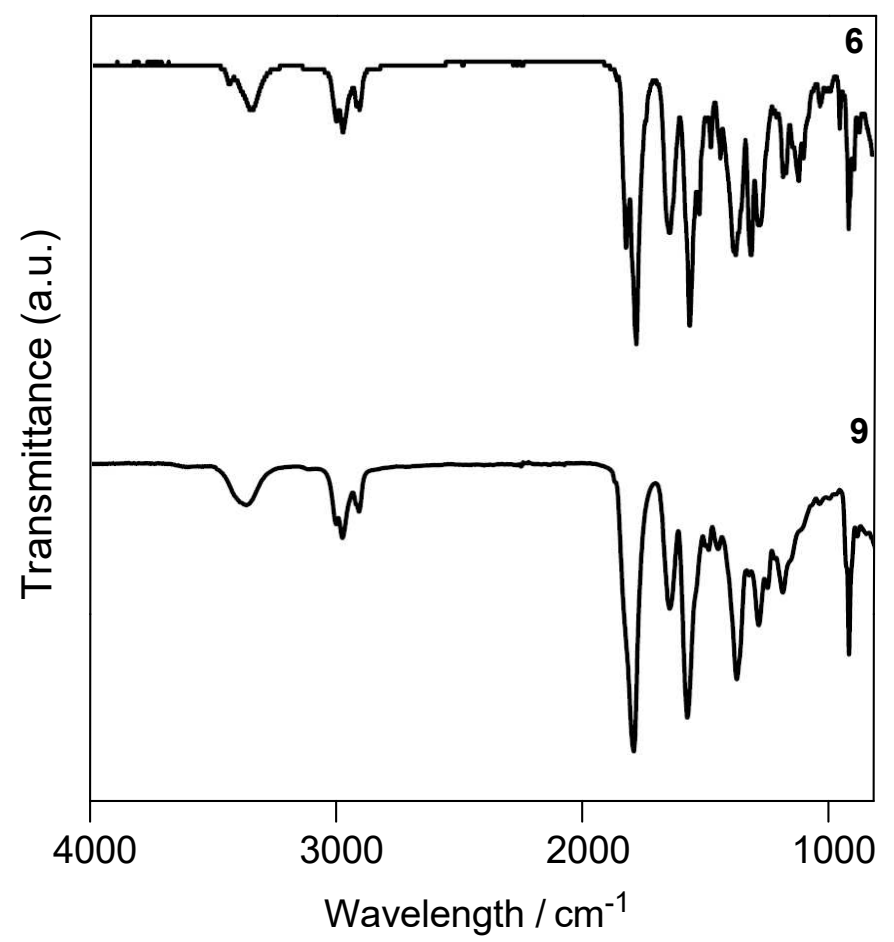

Figure S25. IR spectra of acrylate 6 and polymer 9

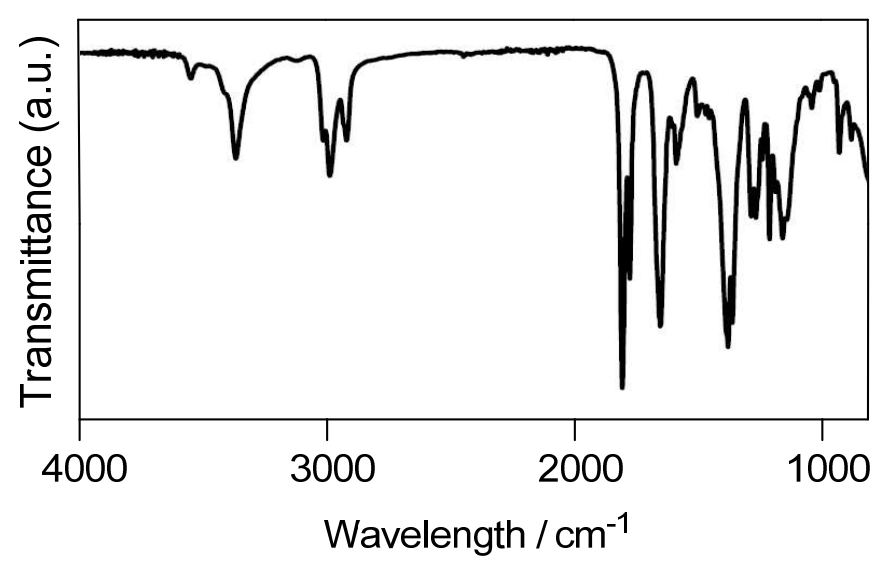

Figure S26. IR spectrum of monool bearing a cyclohexane core 


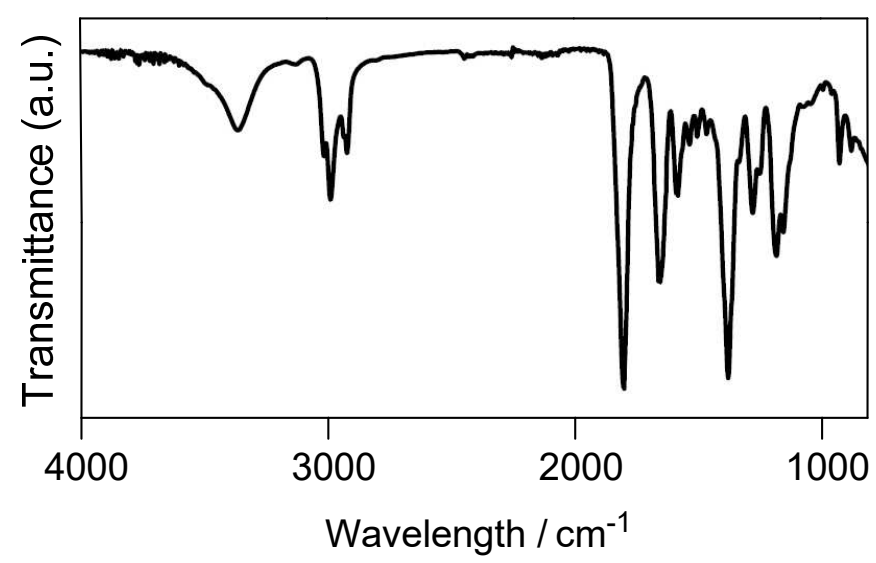

Figure S27. IR spectrum of monool bearing an alkyl moiety

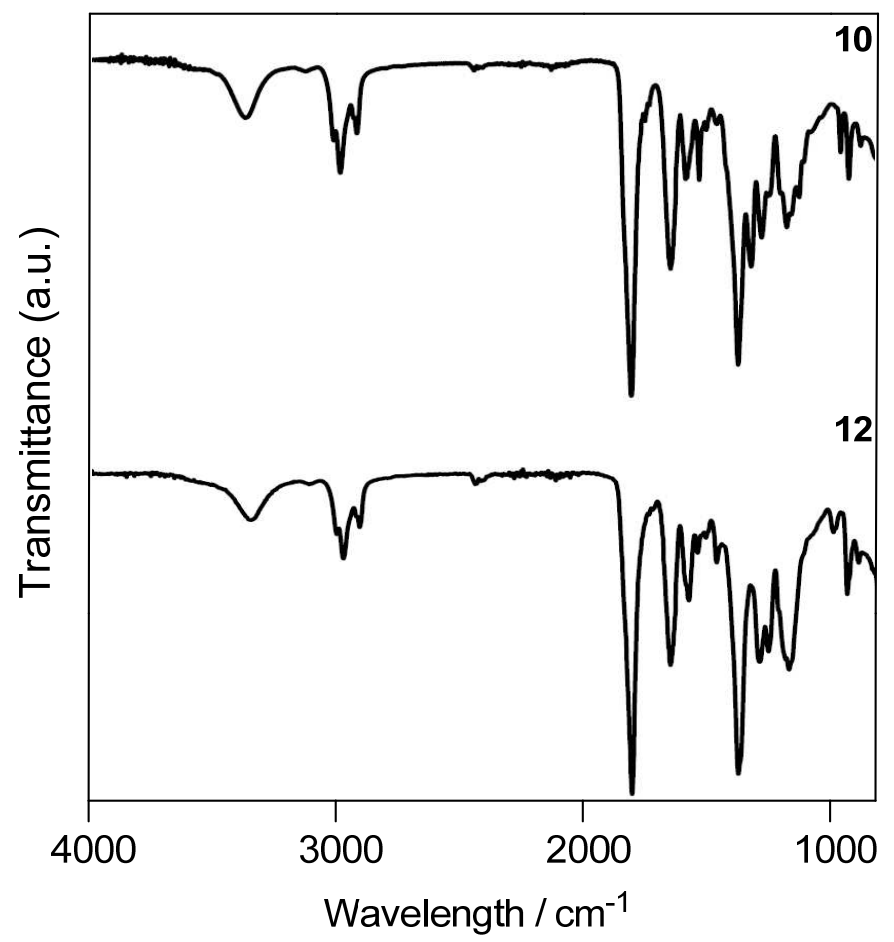

Figure S28. IR spectra of acrylate $\mathbf{1 0}$ and polymer $\mathbf{1 2}$ 


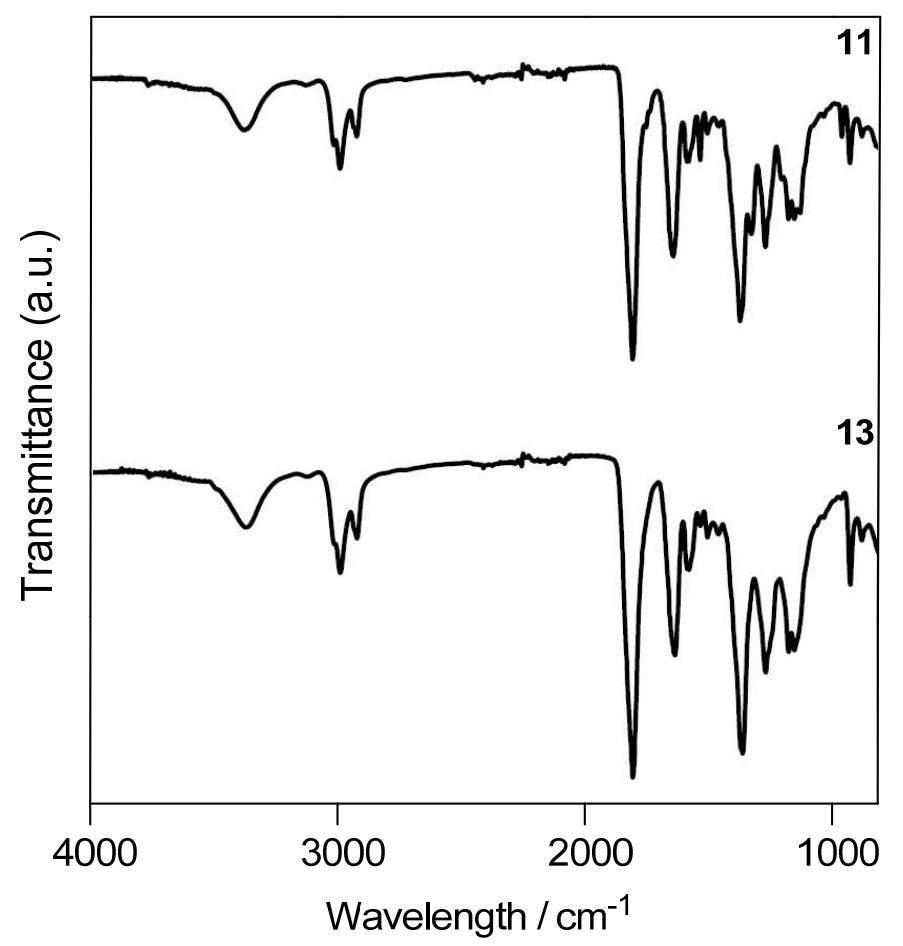

Figure S29. IR spectra of acrylate $\mathbf{1 1}$ and polymer $\mathbf{1 3}$ 


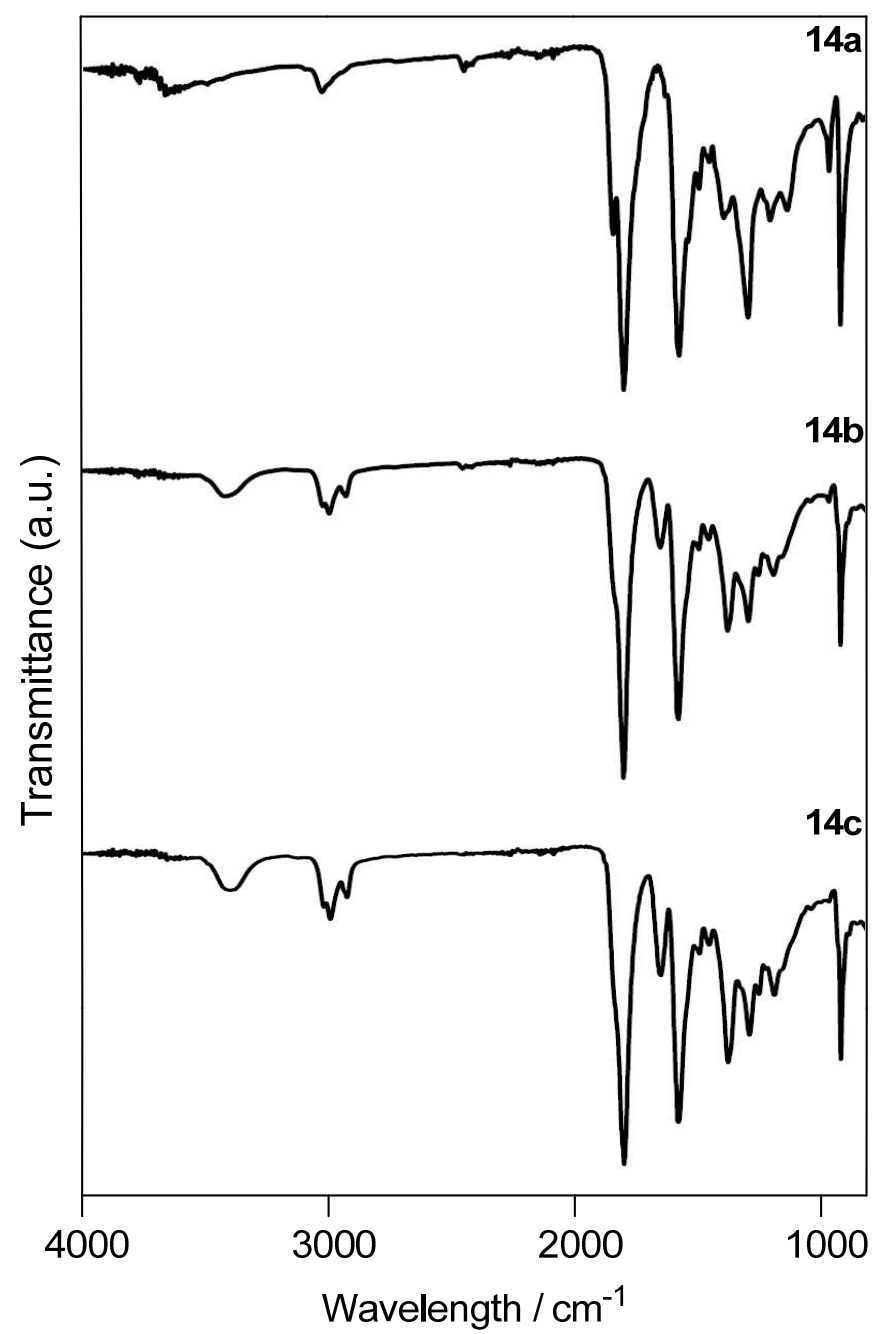

Figure S30. IR spectra of crosslinked polymers 14a-c 


\section{S4. TG data}

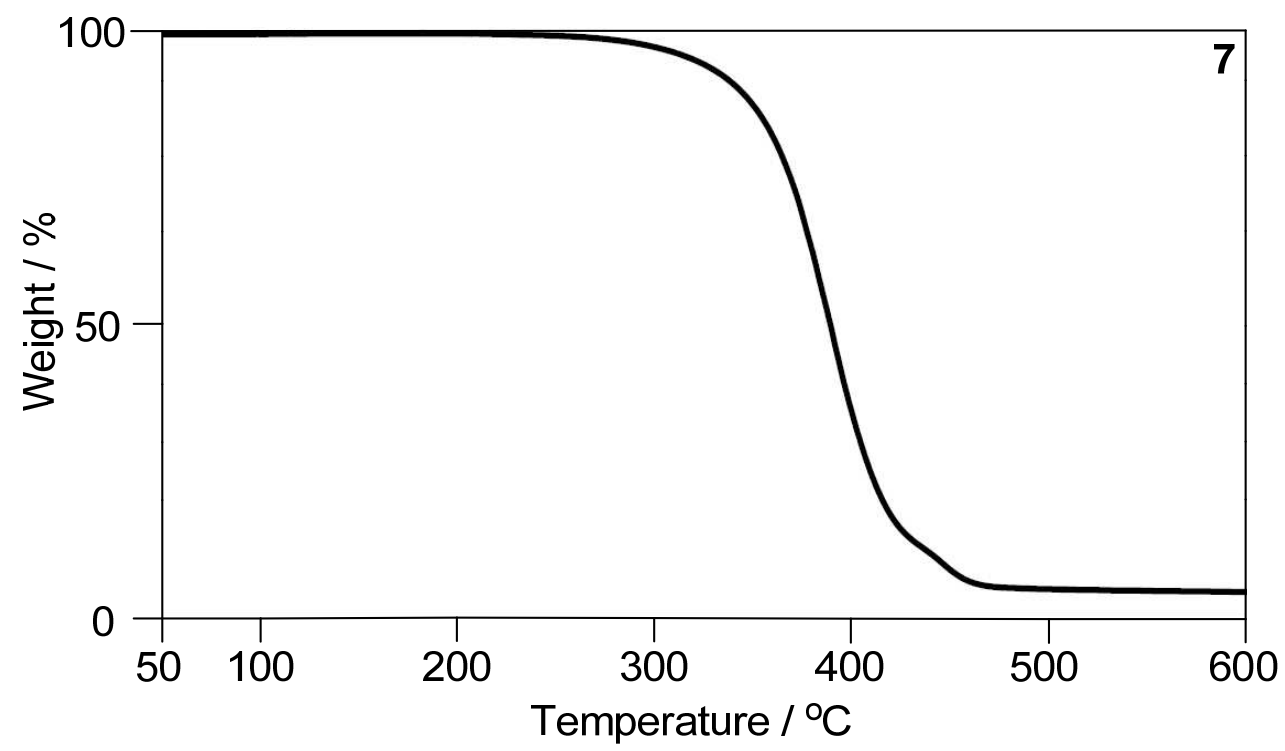

Figure S31. TG-thermogram of polymer 7

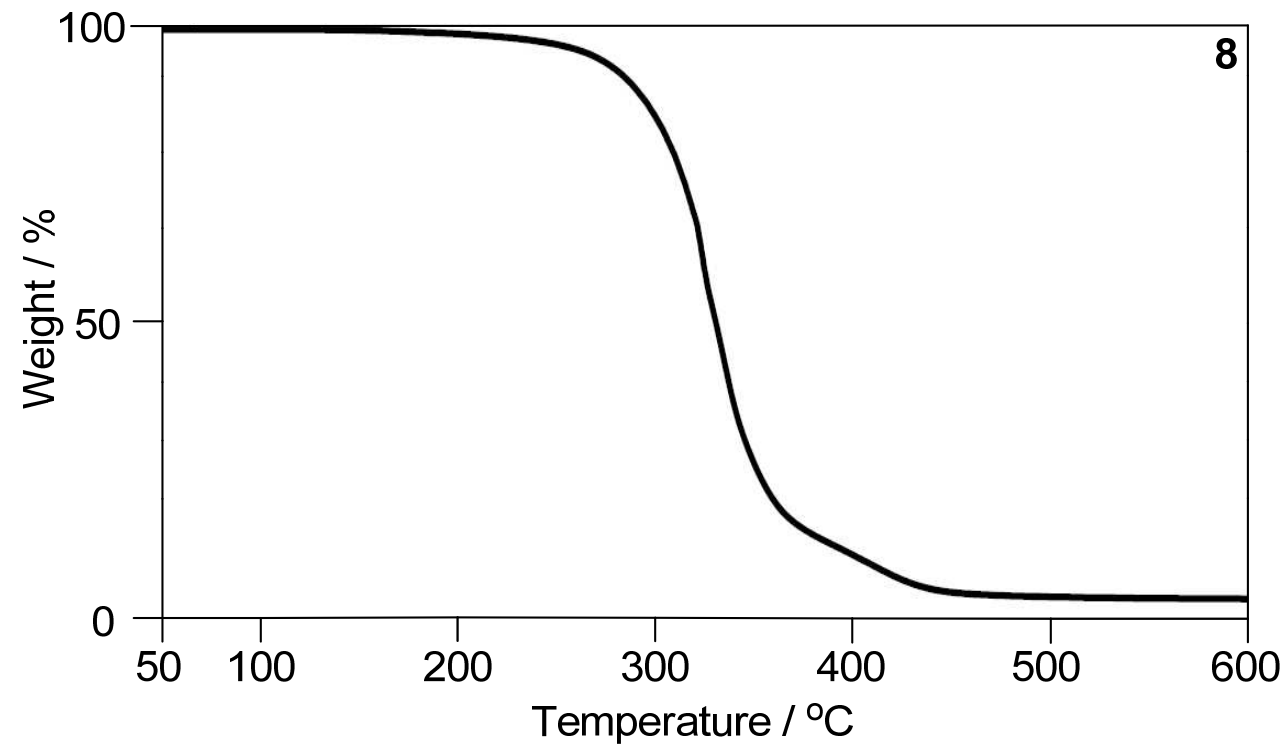

Figure S32. TG-thermogram of polymer $\mathbf{8}$ 


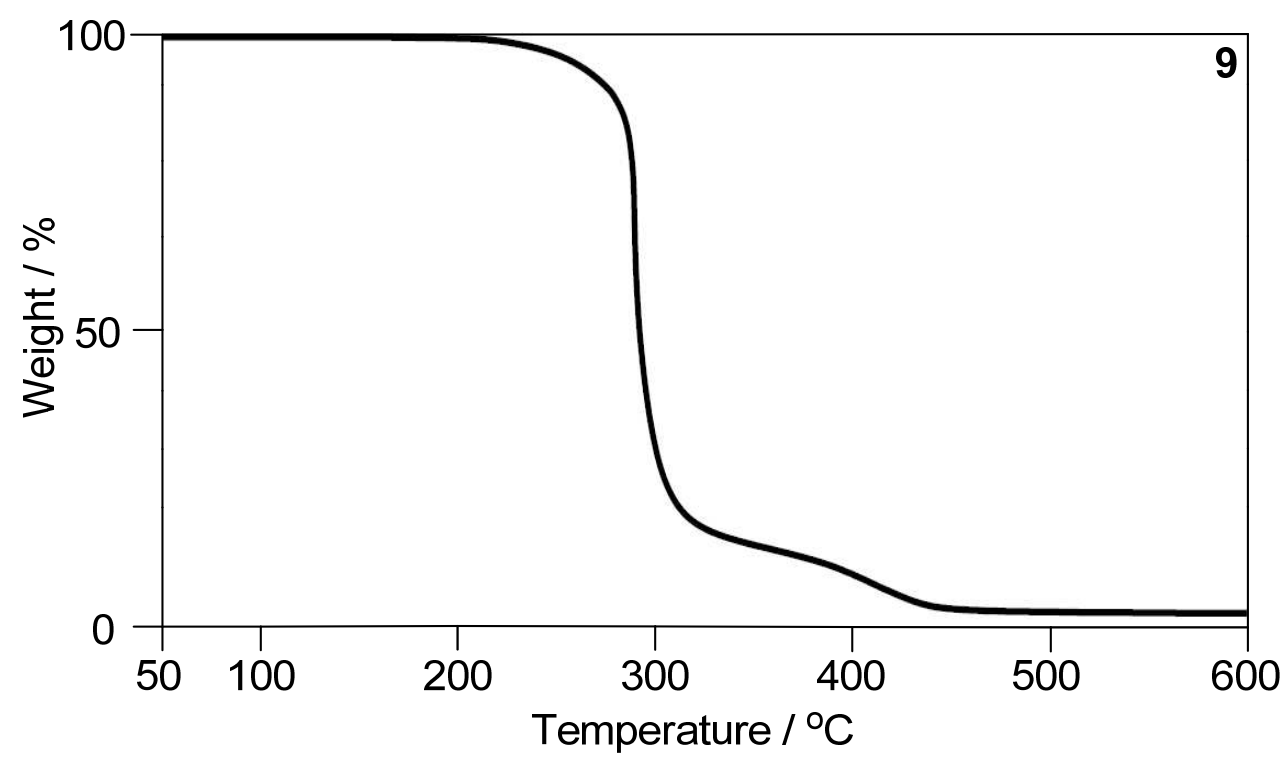

Figure S33. TG-thermogram of polymer 9

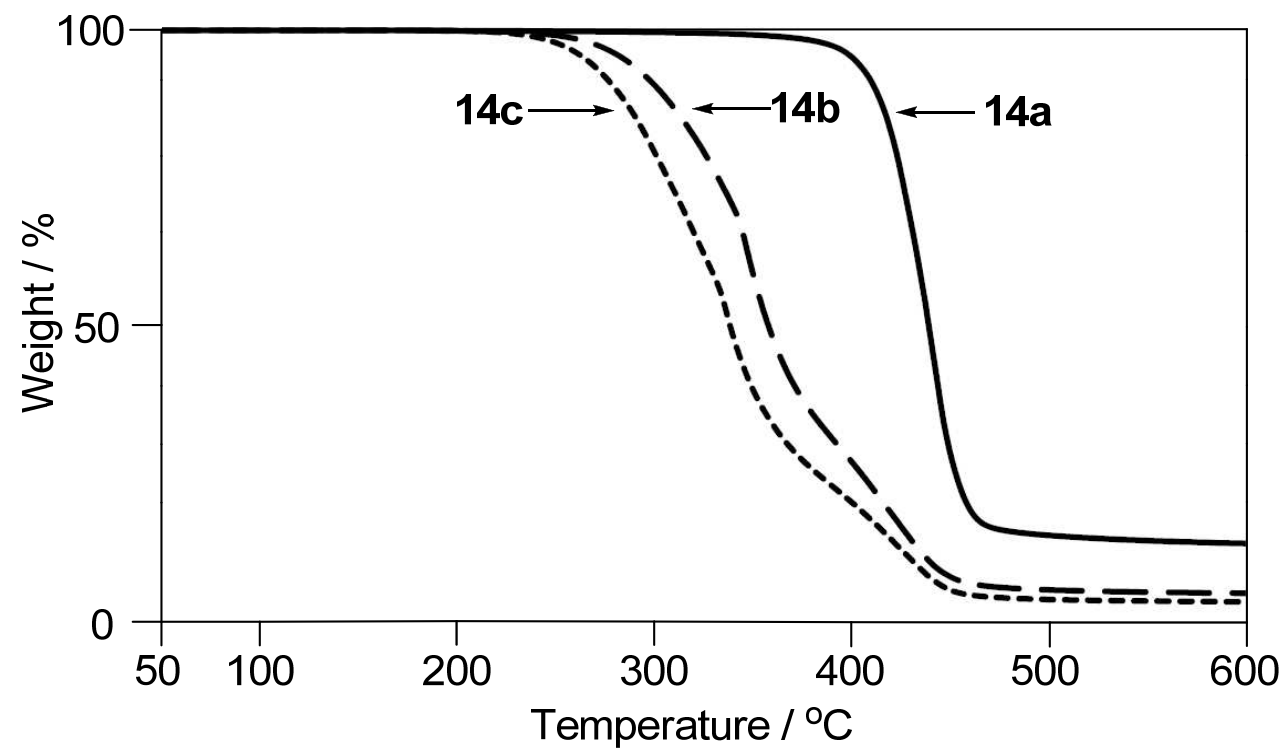

Figure S34. TG-thermograms of crosslinked polymers 14a-c 


\section{S5. DSC data}

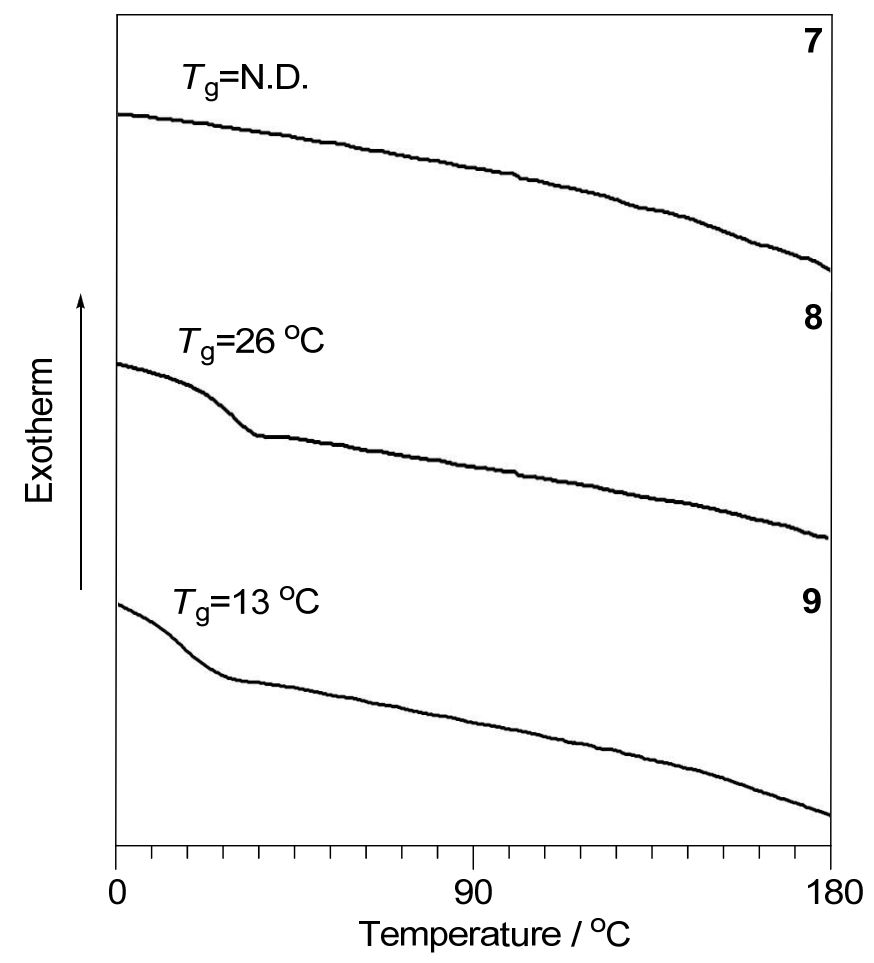

Figure S35. DSC-thermograms of polymers 7-9

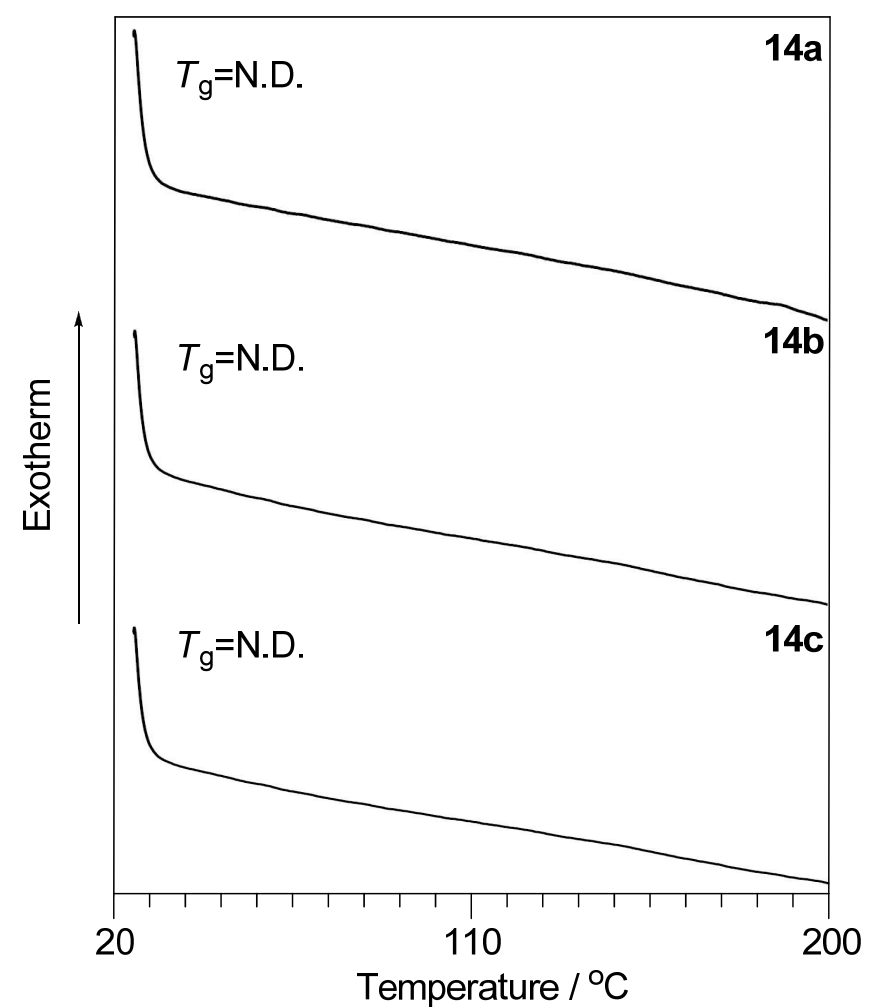

Figure S36. DSC-thermograms of crosslinked polymers 14a-c 
S6. Volume change during radical photo-copolymerization of TAI and 6

Table S1. Results of volume change during radical photo-copolymerization of TAI and 6

\begin{tabular}{|c|c|c|c|c|c|}
\hline Entry & $\begin{array}{c}{[\mathrm{TAI}]_{0}:[6]_{0}} \\
\text { (Feed ratio) }\end{array}$ & $\mathrm{D}_{\text {before }}\left(\mathrm{g} / \mathrm{cm}^{3}\right)^{a}$ & Polymer & $\mathrm{D}_{\text {after }}\left(\mathrm{g} / \mathrm{cm}^{3}\right)^{a}$ & $\begin{array}{c}\text { Volume } \\
\text { Change }(\%)\end{array}$ \\
\hline 1 & $100: 0$ & 1.339 & $\mathbf{1 4 a}$ & 1.365 & -1.94 \\
\hline 2 & $70: 30$ & 1.263 & $\mathbf{1 4 b}$ & 1.276 & -1.03 \\
\hline 3 & $50: 50$ & 1.236 & $\mathbf{1 4}$ & 1.242 & -0.49 \\
\hline 4 & $0: 100$ & 1.179 & $\mathbf{9}$ & 1.182 & -0.25 \\
\hline
\end{tabular}

${ }^{a}$ Measured by a pychnometer at $21{ }^{\circ} \mathrm{C}$ under $\mathrm{He}$ atomosphere. 